\title{
Physical and numerical modelling of tsunami generation by a moving obstacle at the bottom boundary
}

\author{
C. N. Whittaker • R. I. Nokes - H.-Y. Lo • P. L.-F. \\ Liu · M. J. Davidson
}

Received: date / Accepted: date

\begin{abstract}
This paper presents a study of the waves generated by a solid block landslide moving along a horizontal boundary. The landslide was controlled using a mechanical system in a series of physical experiments, and laser-induced fluorescence measurements resolved both spatial and temporal variations in the free surface elevation. During its constant-velocity motion, the landslide transferred energy into 'trapped offshore-propagating waves within a narrow frequency band. The
\end{abstract}

C. N. Whittaker

Department of Civil and Environmental Engineering

University of Auckland

20 Symonds St

Auckland 1010, New Zealand

E-mail: c.whittaker@auckland.ac.nz

R. I. Nokes

Department of Civil and Natural Resources Engineering

University of Canterbury

Private Bag 4800

Christchurch 8140, New Zealand

H.-Y. Lo

School of Civil and Environmental Engineering

Cornell University

220 Hollister Hall

Ithaca, NY 14853, USA

P. L.-F. Liu

School of Civil and Environmental Engineering

Cornell University

220 Hollister Hall

Ithaca, NY 14853, USA

Department of Civil and Environmental Engineering

National University of Singapore

Singapore 117576

Institute of Hydrological and Oceanic Sciences

National Central University

Jhongli, Taoyuan, 320, Taiwan

M. J. Davidson

Department of Civil and Natural Resources Engineering

University of Canterbury

Private Bag 4800

Christchurch 8140, New Zealand 
wave trapping is demonstrated by investigating the wave dispersion characteristics using a twodimensional Fourier Transform. The first of the trailing waves broke at Froude numbers greater than or equal to 0.625 . The parametric dependence of the largest-amplitude waves and the potential energy within the wave field are discussed.

The experimental results were compared to the predictions of an incompressible Navier-Stokes solver with and without turbulence models. The numerical model under-predicted the measured wave amplitudes, although it accurately predicted the measured wave phasing. The turbulent model more accurately predicted the shapes of the trailing waves. Both experimental and numerical results confirmed that investigations into wave generation by submerged objects moving at constant velocity should also consider the initial acceleration of the object, as this affects the overall evolution of the wave field. The applicability of the horizontal-boundary results to more realistic field scenarios is discussed.

Keywords Tsunami · Landslide · Laser-induced fluorescence (LIF) - Computational fluid dynamics

\section{Introduction}

Tsunamis are generated by a variety of impulsive source mechanisms, and are a significant hazard to people and infrastructure in coastal regions around the world. A tsunami source moving in the direction of wave propagation for a period of time can continuously input energy into some of the generated waves. This energy input may amplify the tsunami wave heights, further increasing the hazard posed to coastal communities by these waves.

Horizontal motion of a tsunami source may arise from the segmented rupture of an underwater fault, a landslide, or an underwater volcanic eruption. Tsunamigenic landslides may be either subaerial (initiated above the water surface) or submarine (initiated below the water surface). Submarine landslides, the focus of the current study, are able to mobilise large volumes of sediment over long runout distances $[7,12]$. If such a landslide moves along a relatively mild slope then the landslide is able to input energy into the generated waves over a considerable time period. This creates the possibility of resonance in the extreme case where the landslide velocity is approximately equal to the wave celerity (similar to the Proudman resonance, see Vennell [19]).

Considering the idealised case of a submerged landslide propagating along a horizontal boundary in a two-dimensional domain (requiring the landslide to have effectively infinite width), the possibility of resonance may be determined by calculating the landslide Froude number:

$$
F r=\frac{u_{t}}{\sqrt{g D}}
$$

where $u_{t}$ is the terminal (or maximum) landslide velocity, $g$ is the gravitational acceleration (taken to be constant in this case) and $D$ is the water depth. The high kinetic energy associated with high Froude numbers enables the generation of relatively large waves. A Froude number of unity (1.0) corresponds to a landslide propagating at the shallow water wave speed, leading to resonant amplification of a wave propagating at this speed. This is known as the critical Froude number.

For a landslide propagating through intermediate or deep water, i.e. with $L_{b} / D$ less than the shallow-water limit of 20 [1], the generated waves will propagate as a dispersive group. In nonshallow water resonance occurs when the landslide velocity equals the group velocity of the generated waves. $L_{b}$ is the landslide length used as a characteristic wavelength scale in determining the depth regime above. In reality all landslides have a finite width, such that the generated waves will propagate in two horizontal directions. The lateral spreading of the waves will also reduce the amplification of the waves propagating in the direction of the landslide motion. Additionally, the changing water depth $D$ on even a very mild slope will complicate the conditions for resonance stated in Equation 1. 
A number of studies have investigated the input of energy from a moving landslide into the waves generated by its motion using a combination of physical and mathematical models. Lee et al. [9] conducted a thorough numerical investigation (using the generalised Boussinesq and the forced Korteweg-de Vries equations) into the waves generated by a submerged object moving at a constant velocity in a two-dimensional channel, neglecting the effects of the object acceleration or deceleration. Their model results were compared to measurements from a series of experiments with $L_{b} / D$ values between 13.5 and 18 (thus approaching the shallow-water limit of 20), where a number of resistance wave gauges provided free surface elevation data. Since the physical experiments were conducted in relatively shallow water, the combined effects of nonlinearity and dispersion could only be assessed using their mathematical model. A number of transcritical velocities were tested; subcritical velocities generated a weakly nonlinear and weakly dispersive trailing wave train, while supercritical velocities generated additional upstream-advancing solitary waves. The authors recommended additional experiments to assess the effectiveness of different wave theories in the range $0.2<F r<0.6$.

Tinti and Bortolucci [18] investigated the energy transfer between a submerged landslide and the generated waves in sufficiently shallow water that even weak dispersive effects could be neglected. Their analytical and numerical models predicted the formation of (nondispersive) wave groups propagating in the forward and backward directions. Assuming a long period of landslide motion, the energy within the wave field reached saturation for a non-critical landslide Froude number; this energy saturation was not attained when considering wave propagation in two horizontal dimensions. The steady solution above the landslide was a trough for subcritical Froude numbers and a crest for supercritical Froude numbers, consistent with the analagous open channel flow problem of a uniform flow over a submerged hump. When considering wave generation over a sloping boundary, the authors concluded that the slope did not greatly affect the shape of the generated waves. However, this finding is likely to have been influenced by the shallow-water conditions enforced by their model.

The two-dimensional experiments of Watts [20] modelled a tsunamigenic landslide as a right triangular block sliding down a $45^{\circ}$ slope under the influence of gravity. The mass and initial submergence depth of the landslide were varied during the experiments. The generated waves propagated in the form of an Airy function with a relatively long leading wave followed by a dispersive train. The Ursell number of the landslide determined the relative importance of the near-field nonlinearity, with dispersive effects dominating in the far field.

The effects of an added lateral dimension on the runup and rundown of waves generated by submerged and subaerial rigid landslides were investigated experimentally and numerically by Liu et al. [11]. They determined that the submerged landslide became ineffective at generating waves for submergence depths greater than three times the slide thickness. Enet and Grilli [3] also conducted three-dimensional experiments to investigate wave generation by submerged landslides, although dispersive and nonlinear features could not be independently varied within their setup. From their two- and three-dimensional studies, they concluded that the initial acceleration of the landslide block governed the generated waves, with most of the wave generation occurring during this phase of motion.

Di Risio et al. [2] investigated the waves generated by a rigid block landslide around a conical island in a series of physical experiments, which complemented the analytical solutions of Renzi and Sammarco [14]. Their results demonstrated that edge wave modes can be excited for round coastal geometries, in a similar manner to those excited on a plane beach (Sammarco and Renzi [15]). The characteristics of edge waves generated on a uniform slope were also investigated by Lynett and Liu [13]. Their results confirm that the runup of tsunami waves exhibits strong dependence on the lateral dimension, even if the initial source mechanism can be modelled using only one horizontal dimension (as in this study).

The energy within a wave field generated by a granular subaerial landslide was discussed by Fritz et al. [6], who carried out a wave-by-wave integration of the free surface elevation time series 
measured by a resistance wave gauge (this required the assumption of a propagation velocity for each wave). The use of a pneumatic landslide generator $[4,5]$ permitted much higher Froude numbers than for the majority of submarine landslide investigations, which used gravity forcing only. As demonstrated in the current investigation, control over the motion of a submarine slide will allow a more thorough experimental investigation into the behaviour of the generated waves in the Froude number range suggested by Lee et al. [9].

In the general fluid mechanics problem concerning the propagation of a submerged object over a horizontal boundary, both the initial acceleration and terminal velocity of that object are important factors controlling the dispersive and nonlinear properties of the generated waves. In the experiments of Whittaker et al. [21], the ability to control the motion of an idealised submerged landslide allowed these two parameters to be varied independently. Their experiments used a similar constant-depth approach to Lee et al. [9], but included dispersive effects at intermediate water depths $\left(L_{b} / D\right.$ between 1.43 and 2.86). They found that the generated wave properties were governed by the landslide Froude number. At relatively low Froude numbers $(F r=0.125)$ the wave field was dominated by the offshore- and onshore-propagating waves generated by the initial acceleration of the landslide. At higher Froude numbers $(F r=0.375)$ the landslide could input additional energy into the offshore-propagating waves during its constant-velocity motion, with the initial acceleration (and final deceleration) of the landslide becoming relatively less important for these Froude numbers.

In this paper we present a combined experimental-numerical study of the waves generated by a submarine landslide with Froude numbers between 0.5 and 0.75 . The purpose of the paper is twofold. Firstly, the paper intends to highlight the effects of the solid block motion on the generated waves. In particular, the relative importance of the initial acceleration and the subsequent period of constant velocity are examined, along with the effect of varying the magnitude of this initial acceleration. Secondly, the paper conducts a comparison study with the IHFOAM CFD model. Comparisons against a simpler model (such as the shallow-water models commonly used to predict landslide-generated tsunamis) would be expected to identify issues with the approximations used to formulate the model. A CFD model which directly solves the governing equations of fluid mechanics does not contain the same inherent limitations. As computational capabilities continue to improve, these models are likely to become more commonly applied to predictions of geophysical hazards. The experimental dataset (containing a well-controlled model landslide block and spatial/temporal resolution of the generated waves) is ideal for the validation of such a model.

The physical experiments were conducted using the setup of Whittaker et al. [21], described briefly in Section 2, allowing measurement of the free surface elevation along the entire length of the flume. The experimental results are compared to the predictions of the IHFOAM computational fluid dynamics (CFD) package. The numerical model is described in Section 3, while the experimental measurements are introduced in Section 4. Comparison of the model predictions and experimental measurements in Section 5 demonstrates the ability of the IHFOAM CFD package to accurately capture the experimentally-generated free surface elevations, including instances of wave breaking. Reasons for the small discrepancies between the predicted and measured wave fields are discussed. In Section 6, additional simulations describe the effects of the landslide acceleration in an extended parameter space.

\section{Experimental method}

The experiments used to investigate the waves generated by a submarine landslide were undertaken in a flume of $14.66 \mathrm{~m}$ length and $250 \mathrm{~mm}$ width at the University of Canterbury, New Zealand. The semi-elliptical landslide block (with a thickness of $26 \mathrm{~mm}$ ) moved along a slotted false floor in the central $5 \mathrm{~m}$ of the flume, allowing the maximum time before reflected waves from the flume ends contaminated the wave field. The slot width was $8 \mathrm{~mm}$, and its effects are discussed in Section 5 . 


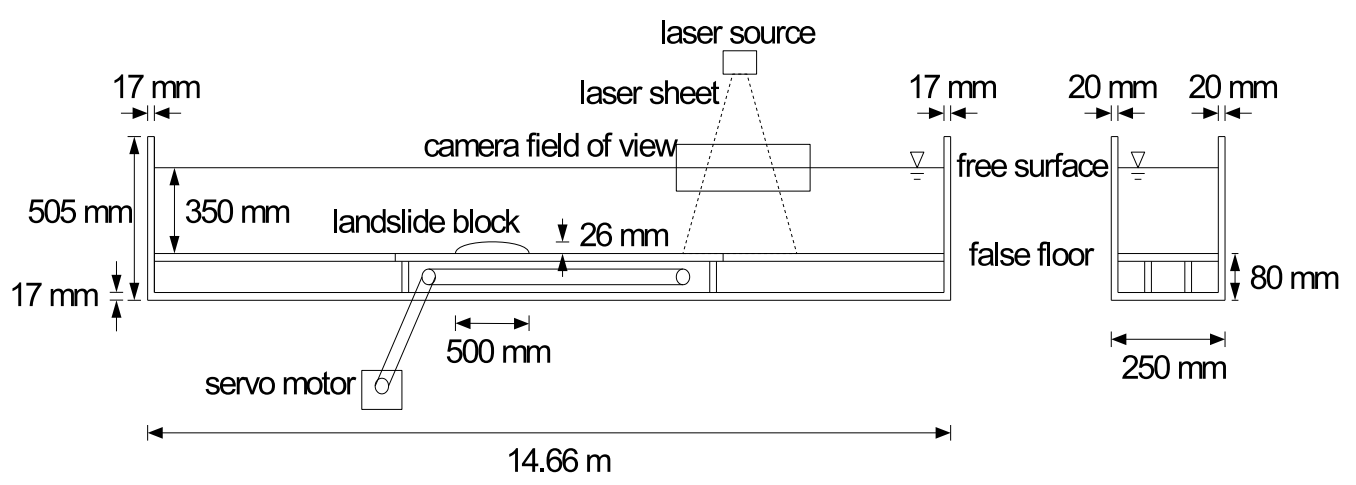

Fig. 1 Experimental setup used by Whittaker et al. [21] to investigate the dependence of wave properties on the acceleration and velocity of a rigid block landslide moving along a horizontal boundary.

Figure 1, from Whittaker et al. [21], shows the experimental setup; the reader is referred to this paper for a more thorough description of the experimental setup and method.

The landslide motion was provided by a servo motor outside of the flume, connected to the landslide by two toothed timing belts. This method of motion control allowed the landslide to achieve its velocity targets to within $\pm 5 \%$ [21]. The landslide motion consisted of an initial period of constant acceleration, a period of constant velocity (at the landslide terminal velocity) and a constant deceleration to rest at the same rate as the initial acceleration. Both the magnitude of the initial landslide acceleration $\left(a_{0}\right)$ and the landslide terminal velocity (represented by the landslide Froude number) were varied during the experiments and simulations.

Section 6 describes the extension of this parameter space within the IHFOAM simulations.

Spatial and temporal variations in the free surface elevation were measured using an application of the laser-induced fluorescence (LIF) technique [21,16]. A small amount of fluorescent dye was added to the water in the flume and illuminated from above by a $532 \mathrm{~nm}$ laser sheet. The dye fluoresced at $545 \mathrm{~nm}$ under the laser excitation, and the interface between the fluorescing dye and the air was recorded using a JAI M4-CL camera outside of the flume (with the laser light filtered out). The camera field of view was $350 \mathrm{~mm}$, and the pixel resolution was $0.305 \mathrm{~mm} /$ pixel. By interpolating between pixels in the vertical direction, the free surface could be identified to within an accuracy of $\pm 0.4 \mathrm{~mm}$. The laser and image acquisition equipment were mounted on a gantry above the flume, which could be positioned accurately using a servo motor; repeating the experiments with different camera locations allowed recording of the free surface variations over the entire length of the flume.

\section{Numerical model}

In this study we use the latest version of IHFOAM (Higuera et al. [8]) to simulate the landslidewave problem numerically. IHFOAM is a variant, designed particularly for coastal engineering applications, of the open-source computational fluid dynamics (CFD) package OpenFOAM. The model adopts a finite volume discretization, and the governing equations are the Reynolds-Averaged Navier-Stokes equations for two-phase flows (water and air). The volume of fluid (VOF) method is used to track the free surface. Various turbulence models are available for use, and the $k-\epsilon$ model was selected for comparisons against the laminar results (i.e. solving the RANS equations using the molecular viscosity and ignoring turbulence). In the subsequent sections of this paper, we refer to the 'laminar' results as those that ignore turbulence and the ' $k-\epsilon$ ' results as those obtained using 


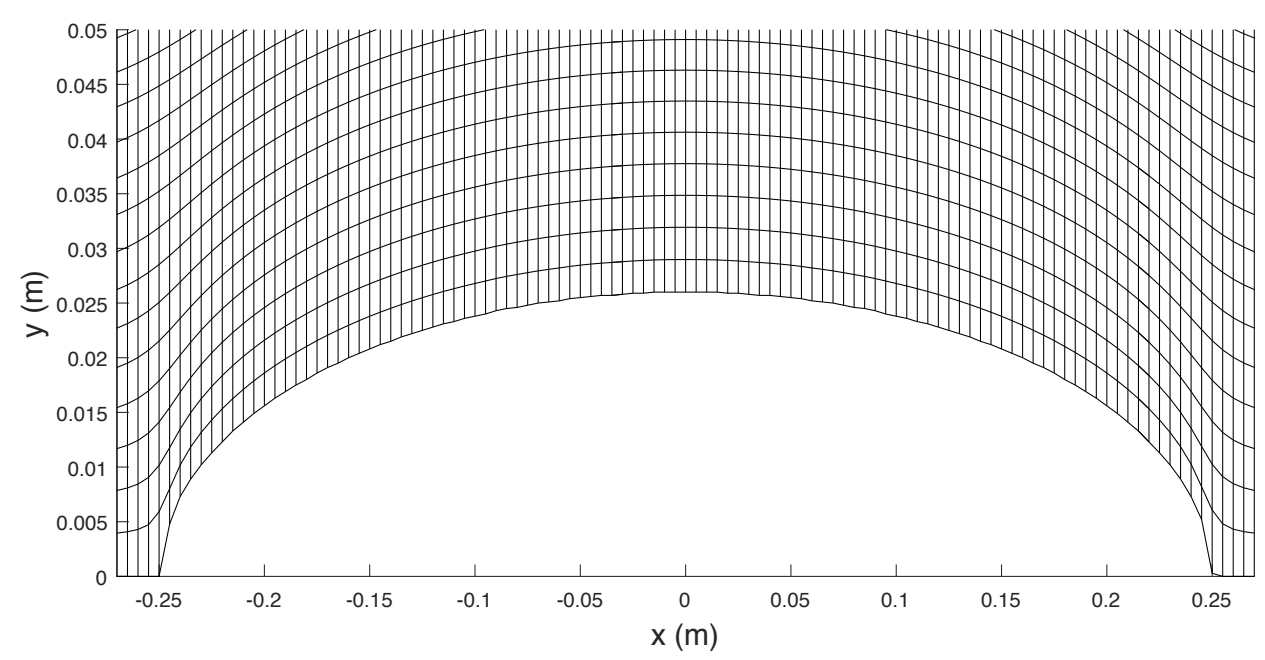

Fig. 2 The deformed mesh due to the semi-elliptical landslide used in this study.

the $k-\epsilon$ turbulence model. More detailed description and validation of IHFOAM can be found in Higuera et al. [8] and their preceding papers.

IHFOAM's ability to easily specify a moving boundary allows us to model the landslide as a moving bottom boundary. The deformation of the boundary is achieved via a dynamic mesh that changes at each time step according to the landslide motion. Therefore, a limitation of this approach is that the landslide shape has to be sufficiently smooth - e.g. a rectangular block with vertical edges cannot be resolved. The deformed mesh due to the semi-elliptical landslide used in this study is shown in Figure 2. The implementation of the numerical wave paddle (moving boundary) in IHFOAM was originally designed to simulate physical wave makers in laboratories. The use of IHFOAM in landslide-wave problems is unprecedented, hence this study also serves as a validation of IHFOAM's ability to simulate water waves generated by a solid landslide.

In the VOF method, the free surface elevation is not directly resolved. Instead, each cell (whose location is represented by the cell center) is assigned a parameter $0 \leq \alpha \leq 1$, with $\alpha=1$ meaning the cell is completely filled with water, and $\alpha=0$ meaning the cell is completely dry. To determine the free surface elevation $\eta$ from CFD data, at each horizontal location $x$ we search for the first y-coordinate where $\alpha=0.5$ by interpolating between cells from top (air) to bottom (water). When wave breaking occurs (Experiments 7 and 8, see Table 1), the water column at each $x$ location may no longer be continuous and in such case $\eta$ cannot be meaningfully defined. Based on our approach, a value is still assigned to $\eta$ at these locations, and it can be loosely interpreted as the height of the highest water droplet at each $x$ location when viewed from above. Note that the $\alpha=0.5$ is a somewhat arbitrary definition of the free surface, and may affect the free surface elevation recorded within the model.

The computational domain mimics the physical laboratory setup - a $14.66 \mathrm{~m}$ long tank with either $0.175 \mathrm{~m}$ or $0.35 \mathrm{~m}$ water depth. The numerical ceiling (atmospheric boundary condition) was placed high enough above the free surface to ensure that no water could come in contact with it. The initial landslide center is at $x=0 \mathrm{~m}$, the rear end of the tank at $x=-4.88 \mathrm{~m}$, and the front end at $x=9.78 \mathrm{~m}$. Solid wall boundary conditions are used at both ends to allow wave reflection, consistent with the physical experiments. It should be noted that the experimental conditions were not perfectly reflective, due to filling taps placed in the ends of the flume. The possibility of flow through the slotted false floor in the offshore section of the experimental wave flume was also not included in the idealised numerical model geometry. 

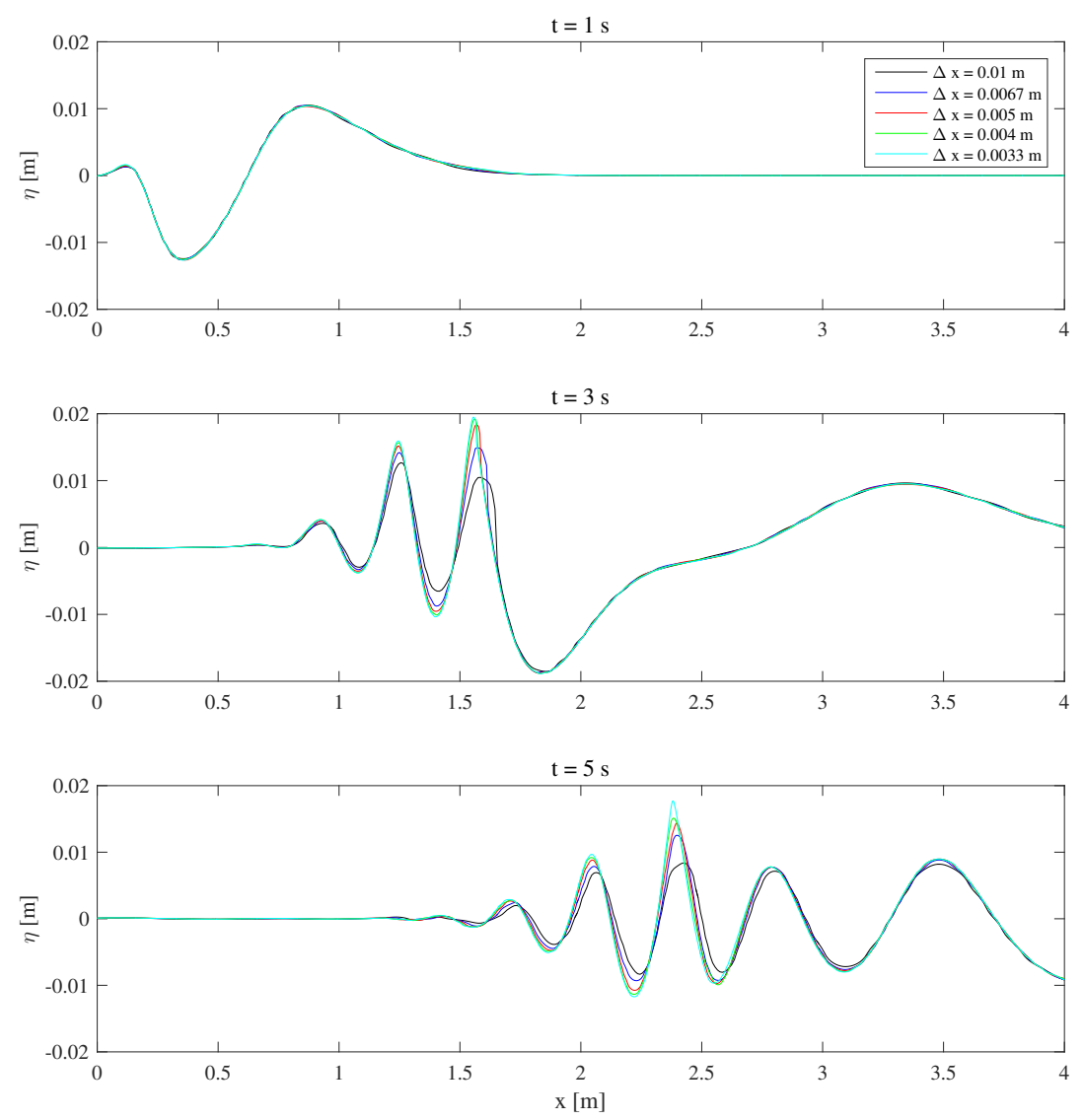

Fig. 3 Dimensional numerical convergence results for the laminar CFD model (i.e. neglecting turbulence), computed with different grid sizes for Experiment 6 at $t=1 \mathrm{~s}, t=3 \mathrm{~s}$ and $t=5 \mathrm{~s}$.

A convergence test based on Experiment 6 (see Table 1) illustrates the effect of grid size on the amplitudes and phasing of the generated waves. Figures 3 and 4 illustrate this at $t=1 \mathrm{~s}, t=3 \mathrm{~s}$ and $t=5 \mathrm{~s}$. In this and other figures, the reader should refer to the online version for the coloured lines. The leading waves exhibited negligible variation with grid size, and may be modelled by even a relatively coarsely-resolved model. However, the short trailing waves (discussed in more detail in Section 4) were very sensitive to the grid resolution. It should be noted that the vertical grid spacing varied, and was finer near the free surface (approximately half the horizontal grid spacing).

The results for the laminar and turbulent models also differed for these waves. In terms of wave amplitudes, the turbulent model appeared to converge much more rapidly than the laminar model at $t=3 \mathrm{~s}$. At $t=5 \mathrm{~s}$, the trailing waves simulated within the laminar model exhibited strong phase and amplitude variation with grid resolution. The reason for this is that the trailing waves broke slightly in the laminar numerical simulations. This breaking behaviour was partially smoothed out by the additional numerical dissipation present in the $k-\epsilon$ model, leading to the phase shifts observed in Figure 4. Further discussion on the CFD model's ability to correctly predict the relatively short and steep trailing waves is provided in Section 5 . 

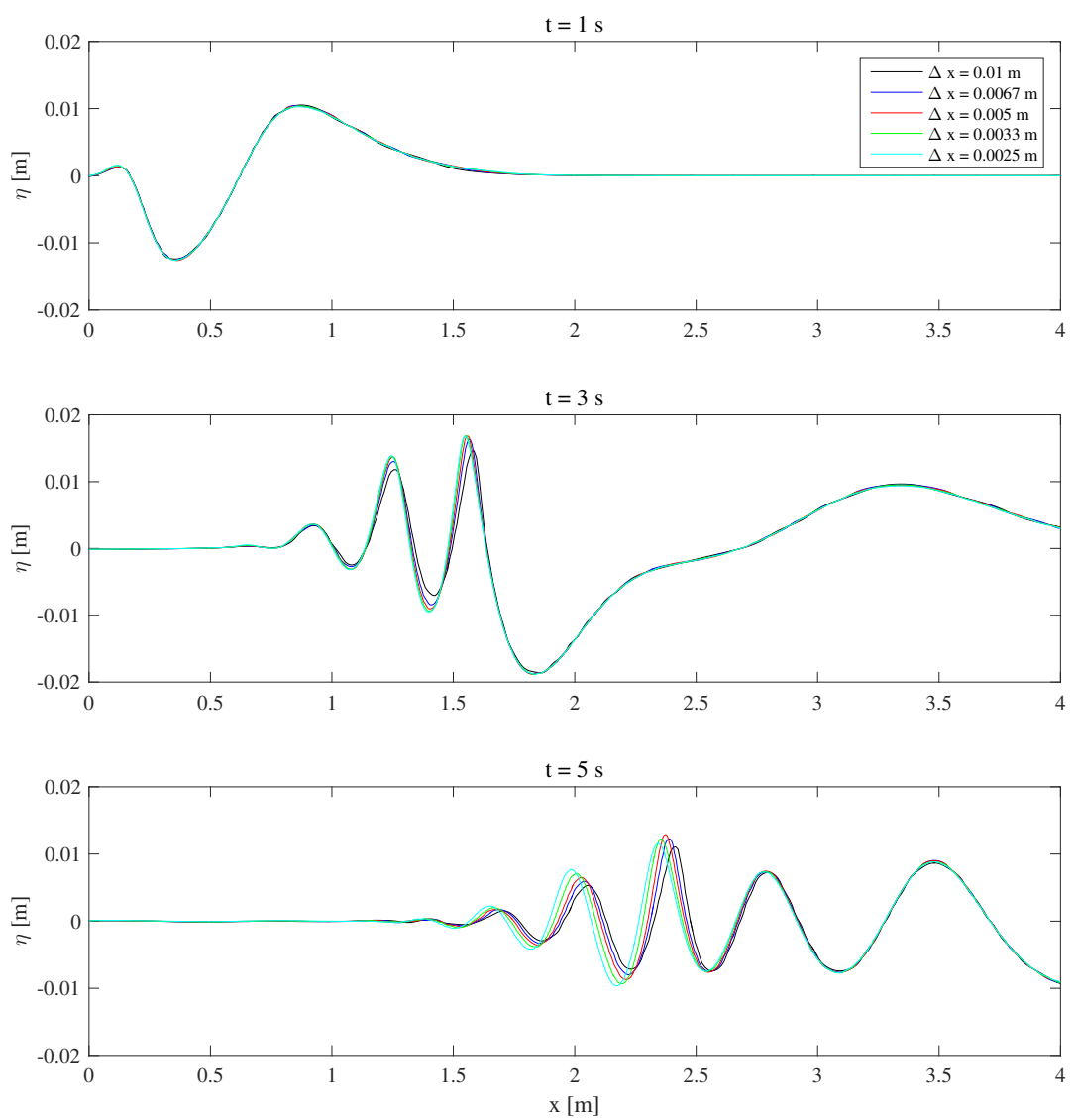

Fig. 4 Dimensional numerical convergence results for the $k-\epsilon$ CFD model (i.e. including turbulence), computed with different grid sizes for Experiment 6 at $t=1 \mathrm{~s}, t=3 \mathrm{~s}$ and $t=5 \mathrm{~s}$.

The convergence rate of the numerical results is examined, by plotting the root mean square difference of the surface elevation as a function of grid size. The VOF surface tracking method is first-order accurate, so without the k-e model we would expect to see a linear convergence rate plot, which is indeed the case (see Figure 5). Thus, we can conclude that our numerical results have converged (we have reached the theoretical maximum rate of convergence). A resolution of $\Delta x=0.005 \mathrm{~m}$ was used in all subsequent simulations.

\section{Experimental wave field properties}

4.1 Nondimensional parameter space examined during the physical experiments

As with many physical laboratory studies of environmental flows, the laboratory measurements are most readily interpreted in a nondimensional form. This allows easier comparison of the mea- 

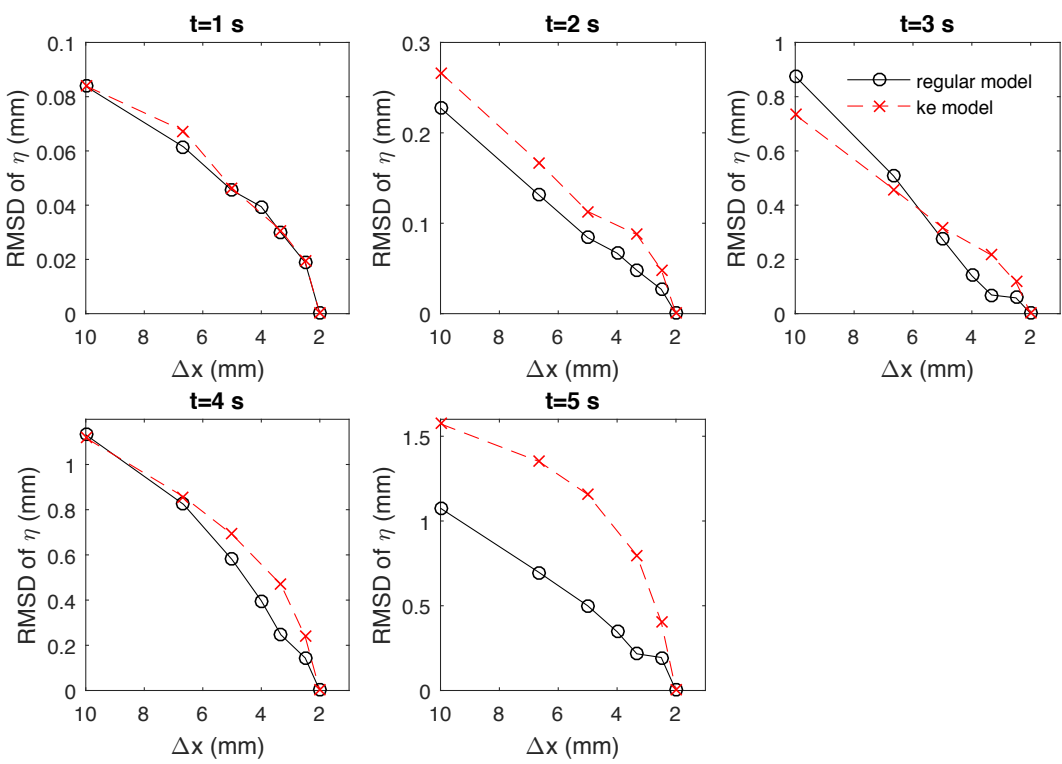

Fig. 5 Numerical results computed with different grid sizes for Experiment 6.

surements to the numerical model results, and aids the interpretation of the results at field scales. Following Whittaker et al. [21], the nondimensionalised experimental variables are:

$$
x^{\prime}=\frac{x}{L_{b}}, y^{\prime}=\frac{y}{L_{b}}, t^{\prime}=t \sqrt{\frac{g}{L_{b}}}, \eta^{\prime}=\frac{\eta}{h_{b}},
$$

where the horizontal distance along the flume $x$ has the origin above the initial location of the landslide centre of mass, $y$ is the vertical coordinate within the flume (with its origin at the free surface), $t$ is the time relative to the start of the landslide motion, $\eta$ is the free surface elevation, $g$ is the gravitational acceleration, $L_{b}$ is the landslide block length $(500 \mathrm{~mm})$ and $h_{b}$ is the landslide block thickness $(26 \mathrm{~mm})$. In general, the landslide thickness is used to nondimensionalise the perturbations (such as the wave elevation $\eta$ ), while the landslide length is used to nondimensionalise other length scales (such as $x$ and $y$, although $y$ could have just as easily been nondimensionalised by the water depth $D$ ). Nondimensional variables will be used throughout the remainder of this paper (with primes omitted for convenience), unless otherwise stated.

The nondimensional framework admits three useful parameters for characterising the different experiments:

$$
\tau=\frac{D}{L_{b}}, \quad \lambda=\frac{a_{0}}{g}, \quad F r=\frac{u_{t}}{\sqrt{g D}}
$$

where $\tau$ is the nondimensional water depth (determining the likely importance of dispersion for the generated waves), $\lambda$ is the nondimensional landslide acceleration and $F r$ is the landslide Froude number (repeated here for convenience). The deep and shallow water limits are therefore $\tau \leq 2$ and $\tau \geq 20$ respectively. Note that although the landslide length scale is used to nondimensionalise the length and time-scales of the landslide motion, the landslide Froude number (in its standard form) uses the depth as its length scale. Since the speed of a shallow water wave in a channel of depth $D$ is $\sqrt{g D}$, this form of the Froude number has more physical meaning. 
Table 1 Nondimensional parameters for high-Froude number experiments and simulations

\begin{tabular}{rrrrrrr}
\hline Experiment no. & $F r$ & $\lambda$ & $\tau$ & $t_{1}$ & $t_{2}$ & $t_{3}$ \\
\hline 1 & 0.500 & 0.051 & 0.70 & 8.21 & 17.07 & 25.27 \\
2 & 0.500 & 0.051 & 0.35 & 5.80 & 14.66 & 20.47 \\
3 & 0.500 & 0.102 & 0.70 & 4.10 & 12.96 & 17.07 \\
4 & 0.500 & 0.102 & 0.35 & 2.90 & 11.76 & 14.66 \\
5 & 0.500 & 0.153 & 0.70 & 2.74 & 11.60 & 14.33 \\
6 & 0.500 & 0.153 & 0.35 & 1.94 & 10.79 & 12.73 \\
7 & 0.625 & 0.153 & 0.35 & 2.42 & 11.28 & 13.70 \\
8 & 0.750 & 0.153 & 0.35 & 2.90 & 11.76 & 14.66 \\
\hline
\end{tabular}

Table 1 lists the nondimensional parameters tested in the physical experiments and numerical simulations, where $t_{1}, t_{2}$ and $t_{3}$ are the nondimensional times of motion change from constant acceleration to constant (terminal) velocity, constant velocity to constant deceleration and constant deceleration to rest, respectively. The three nondimensional landslide accelerations used in these experiments were $\lambda=0.051,0.102$ and 0.153 , corresponding to dimensional accelerations of $0.5 \mathrm{~m} / \mathrm{s}^{2}, 1.0 \mathrm{~m} / \mathrm{s}^{2}$ and $1.5 \mathrm{~m} / \mathrm{s}^{2}$ respectively (these accelerations were dictated by the capabilities of the motor described in Section 2). The time to terminal velocity $t_{1}$ was determined by the nondimensional acceleration and the landslide Froude number. The landslide moved at its terminal velocity for $2 \mathrm{~s}$ in each experiment (thus, the value $t_{2}-t_{1}$ was the same for all experiments). The time period for the landslide deceleration was the same as the time of the initial acceleration.

Experiments 1 to 6 are used to investigate the wave field properties at a landslide Froude number of 0.5. Experiments 7 and 8 show the effect of increasing the landslide Froude number. These higher landslide Froude numbers increased the energy input by the landslide into the offshorepropagating waves, causing some of these waves to break. This section will first describe the wave field generated within Experiment 6 (including a repeat experiment with a long constant-velocity phase of motion) before discussing the wave field variation over the entire parameter space. The wave field properties observed at lower Froude numbers are reported in Whittaker et al. [21]; these results will be included in this paper's analysis to provide additional context to the current dataset as required.

\subsection{Experimental wave field properties for a landslide Froude number of 0.5}

Figure 6 shows the wave field measured during Experiment 6, with the parameters $F r=0.5$, $\lambda=0.153$ and $\tau=0.35$. The wave field is presented as a contour plot, displaying its evolution along the length of the flume (the horizontal axis, with the landslide moving in the positive direction) and in time (the vertical axis). The colour scale represents the amplitude of the generated waves, with wave crests and troughs displayed as light and dark bands respectively. With reference to the nondimensionalisation of Equation 2 and the $26 \mathrm{~mm}$ landslide thickness, the maximum amplitude of 0.85 corresponds to a maximum dimensional amplitude of approximately $22 \mathrm{~mm}$. The dashed horizontal lines on the figure indicate the times of motion change $t_{1}, t_{2}$ and $t_{3}$ (defined in the previous section), while the solid red line shows the location of the landslide centre of mass as a function of time (based on the landslide control system described by Whittaker et al. [21]). The dash-dot lines show the characteristic curves of a wave travelling from the origin at the shallow water wave speed.

The acceleration of the landslide along the horizontal boundary generated waves in both the onshore (negative $x$ ) and offshore (positive $x$ ) directions, each group consisting of a long leading wave followed by a dispersive train $[9,20,17]$. The leading wave propagated at approximately the shallow water wave speed (shown by the dash-dot lines in Figure 6), as shown in Figure 6 . The wave field shown in Figure 6 is clearly dominated by the offshore-propagating waves, in particular those 


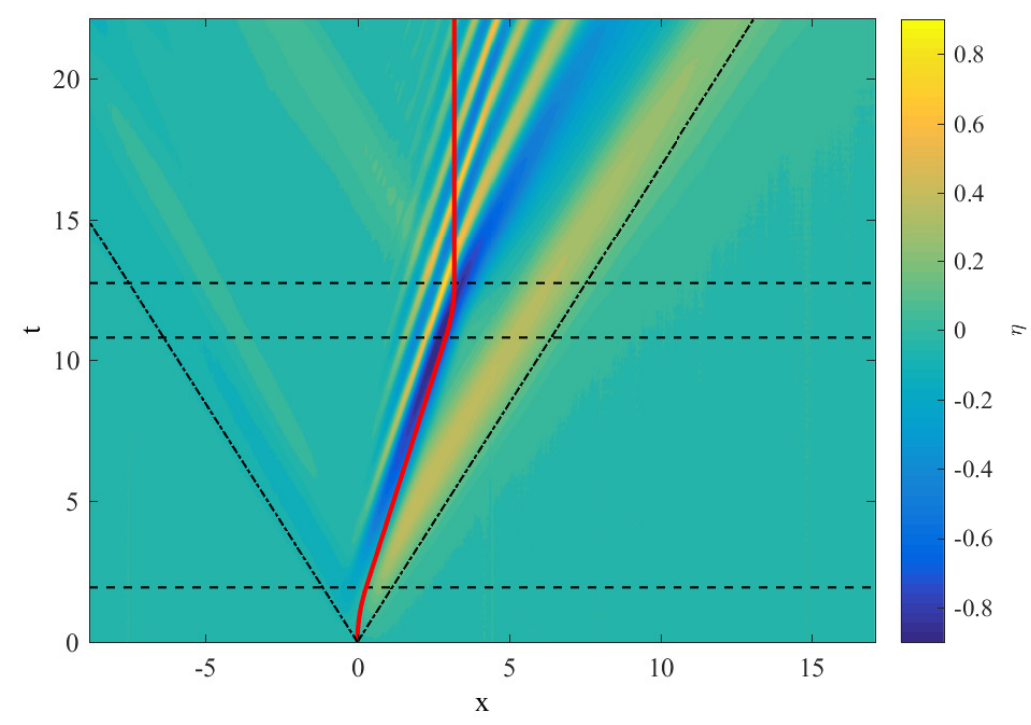

Fig. 6 Wave field observed for Experiment 6, generated by a submerged landslide moving with an acceleration of $0.153 \mathrm{~g}$, a Froude number of 0.5 and a nondimensional water depth of $0.35(175 \mathrm{~mm})$, with the landslide motion shown as a solid red line and the times of motion change shown as dashed horiztonal black lines. The dash-dot lines show the characteristic curves of a wave travelling from the origin at the shallow water wave speed.

waves within the dispersive train propagating with a celerity $c_{p}$ approximately equal to the landslide terminal velocity $u_{t}$. Although waves were initially generated by the landslide acceleration, these were significantly modified by the constant-velocity motion of the landslide. Additional waves were generated by the constant-velocity landslide motion with $c_{p}=u_{t}$, see Lighthill [10]. A free surface depression was generated above the landslide during its constant-velocity motion. This depression had a larger amplitude than the troughs located within the dispersive wave train; the amplitude of the leading onshore-propagating trough was negligible by comparison.

The deceleration of the landslide generated additional wave groups (long leading waves followed by a dispersive train) of opposite phase to those generated by its initial acceleration. The cessation of the landslide motion also released the free surface depression as an offshore-propagating trough. The effect of the constant-velocity motion is still evident in the dispersive wave train, where those waves with approximately $c_{p}=u_{t}$ had the largest amplitudes after the landslide decelerated to rest.

The preferential input of energy into a narrow wavelength band by the constant-velocity landslide motion was investigated within a series of experiments where the landslide deceleration did not occur during the period of free surface measurement (i.e. $t_{2}>22.15$ ). Figure 7 shows the effect of removing the landslide deceleration from Experiment 6 (shown in Figure 6). As expected, the landslide (moving in the offshore direction) imparted more energy into the offshore-propagating waves than the onshore-propagating waves during its acceleration phase. However, the vast majority of the energy in the offshore propagating group was contained within waves propagating at the same speed as the landslide. These waves occupied a region between the solid red line (above the landslide centre of mass, and hence the free surface depression) and the dotted black line in Figure 7. The dotted black line represents the group velocity, assumed to be $c_{g}=0.5 c_{p}$ for these relatively short waves. The growth of the trailing wave group is consistent with the forcing (i.e. landslide) travelling more rapidly than the group velocity of the generated waves. If the landslide terminal velocity was less than even the group velocity of the shortest waves within the dispersive group, it 


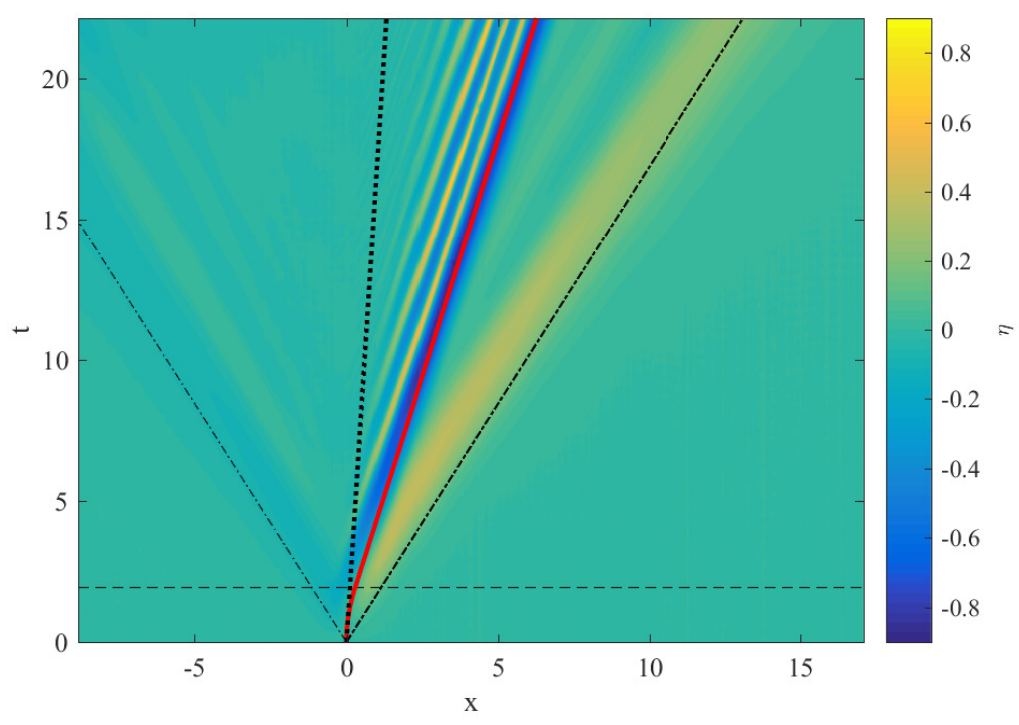

Fig. 7 Wave field generated by a submerged landslide using the same parameters as in Figure 6 (Experiment 6 , with $\lambda=0.153, F r=0.5$ and $\tau=0.35$ ), but with no deceleration during the measurement period. The time of motion change from constant acceleration to constant velocity is shown by a horizontal dashed line, the solid red line shows the position of the landslide centre of mass and the dotted line shows the trajectory of a body travelling at $50 \%$ of the landslide velocity.

would be left behind by the group and would only generate the free surface depression during its constant-velocity motion.

The input of energy by a submerged object moving at constant velocity is reasonably well known (see Lighthill [10] for the problem of a submerged object in a stream). However, the final form of the wave field clearly depends on both the landslide terminal velocity and the wave group generated by the landslide acceleration. The amplitudes within the group increase with the magnitude of the landslide acceleration, but decrease with increasing submergence depth, while the wavelengths of the group increase with the submergence depth [21]. Although the energy distribution among the different wavelengths of the group would presumably change by varying the shape of the landslide acceleration profile (or the shape of the landslide itself), this effect was not investigated in the current project. However, the acceleration parameter space was extended within the simulations described in Section 6.

The effect of the landslide on the dispersion relation of the generated waves (i.e. which waves were trapped and propagated with the landslide velocity) is best observed as a function of the landslide Froude number. Whittaker et al. [21] discussed the effect of landslide Froude numbers between 0.125 and 0.375 on the properties of the generated wave field, and how this parameter influenced the time-varying integrated quantities of the wave field. In this section of the paper, we combine the $F r=0.5$ data presented in this paper with their data to illustrate the effect of the landslide Froude number on the trapped and freely-propagating wave content within the generated wave field. The effect of landslide Froude number, acceleration and submergence depth on the maximum free surface elevations within the wave field are also discussed in Section 4.4.

The relative importance of the waves generated by the initial landslide acceleration and the 'trapped' waves propagating with the landslide during its constant-velocity motion are illustrated for four different Froude numbers in Figure 8, which shows the two-dimensional amplitude spectra from a (dimensional) 2D-FFT (Fast Fourier Transform) of the offshore-propagating waves. Four 

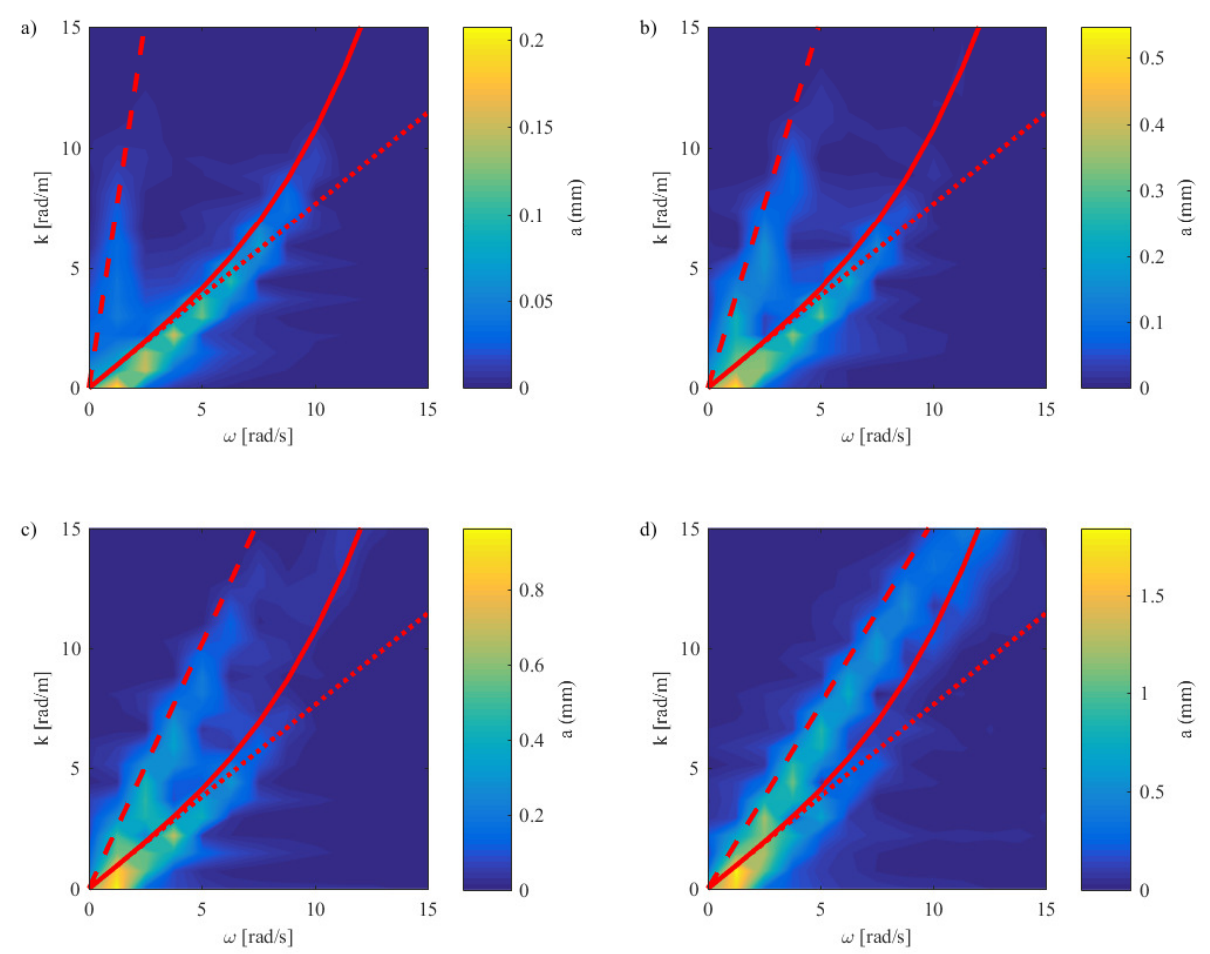

Fig. 8 Two-dimensional wave spectra (from 2D-FFT) for the offshore-propagating waves generated by a landslide with $\lambda=0.153, \tau=0.35$ and a) $F r=0.125$, b) $F r=0.25$, c) $F r=0.375$ and d) $F r=0.5$. The solid line represents the linear dispersion relation, the dotted line represents the shallow-water wave speed and the dashed line represents the landslide velocity.

different landslide Froude numbers are considered: 0.125, 0.25, 0.375 and 0.5. All of the experiments were conducted with the same landslide acceleration $(\lambda=0.153)$ and submergence depth $(\tau=0.35)$. The deceleration of the landslide did not occur while measurements were being recorded, so the landslide motion consisted of an initial acceleration followed by a long period of constant velocity. Thus, the wave field for the $F r=0.5$ case is illustrated in Figure 7 (the lower-Fr cases are discussed in Whittaker et al. [21]).

The restriction of the analysis to the offshore-propagating waves (i.e. the positive $x$-domain) ensures that all of the waves are propagating in the same direction, a requirement for the direct 2D-FFT application. For the same reason, in these experiments the deceleration to rest did not occur during the measurement period (i.e. the first 5 seconds after the initiation of the landslide motion).

In Figure 8, the solid line represents the linear dispersion relation between the wave angular frequency, $\omega$, and the wave number $k$, and the dotted line represents the shallow-water limit of the dispersion relation. The dashed line represents the landslide velocity. The figure clearly illustrates the increasing importance of the trapped waves, or waves propagating at the landslide velocity rather than at their free wave speed (according to linear dispersion), with increasing landslide Froude number. At the lowest Froude number of 0.125 , the free surface depression above the landslide during its constant-velocity motion was very small, and the majority of the waves propagated 
freely after being generated by the initial acceleration of the landslide. The largest-amplitude waves at this Froude number propagated at approximately the shallow-water wave speed. As the Froude number increased, the relative importance of these freely-propagating waves decreased, although the largest waves still propagated at approximately the shallow-water wave speed. At the Froude number of 0.5 , the spectra were dominated by those waves propagating at the landslide velocity.

The non-zero amplitude of the $k$-omega spectrum at $k=0$ is a consequence of the consideration of only the offshore (positive $x$ ) region of the experimental domain. During the period of landslide motion, the offshore-propagating waves were responsible for positive mass transport, such that $\int \eta(x, t) d x>0$ in the offshore region of the domain [21]. This mass transport was predicted by a linear wave model, and may itself be explained by the movement of the landslide volume from its initial position with centre of mass at $x=0$ to the offshore region (such that half of the volume initially located in the onshore region moved into the offshore region). The onshore-propagating waves were responsible for negative mass transport of equal magnitude to the positive offshore transport, such that conservation of mass $\left(\int \eta(x, t) d x=0\right)$ was satisfied over the entire domain. The positive value of $\int \eta(x, t) d x$ in the offshore region is responsible for the non-zero value of the $k$ - omega spectrum at $k=0$.

For the purposes of comparison, Figure 9 illustrates the same 2D-FFT results for the onshorepropagating waves. In this case, the trapped waves observed in the Fourier decomposition of the offshore-propagating wave field are completely absent over the entire range of Froude numbers tested in the physical experiments. The entire $k-\omega$ spectrum consists of waves satisfying the dispersion relation, which is well-approximated by the shallow-water wave equation at low wave numbers. Thus, a comparison between Figures 8 and 9 illustrates the effect of the ongoing interaction between the landslide and the generated waves, and in particular the effect of the landslide on the dispersion characteristics of those waves. One interesting point of comparison is that the offshore-propagating results of Figure 8 show a slight shift away from the predicted dispersion relation. This same shift is not so prominent in the onshore-propagating results of Figure 9, implying that a transfer flow through the slotted false floor may affect the offshore wave propagation. This effect is further discussed in the context of experimental/numerical comparisons of Section 5.

\subsection{Effect of varying acceleration and submergence depth}

As discussed in Section 1, landslide-generated tsunamis are often modelled by either considering only the initial landslide acceleration or by neglecting the acceleration and considering only wave generation at terminal velocity. Both phases of motion are important, and the generated wave properties will also depend on the landslide submergence depth. This section discusses the effects of varying the landslide acceleration and submergence depth on the wave field properties. Further discussion on Froude number effects is deferred to Section 4.4

Figure 10 shows the evolution of the waves generated by a submerged landslide with a Froude number of 0.5 in the contour plot format of Figure 6 . The rows represent the different accelerations $\lambda=0.051,0.102$ and 0.153 , while the two columns represent the two different submergence depths $(\tau=0.70$ and 0.35$)$.

The reduction in water depth from $\tau=0.70$ to 0.35 ( $D=0.35 \mathrm{~m}$ to $0.175 \mathrm{~m}$ in dimensional terms) leads to the expected increase in wave amplitudes, due to the increased size of the perturbation (landslide thickness) relative to the water depth. The decrease in wavelengths with the decrease in water depth is also expected for these intermediate-depth experiments. In the shallow-water limit, the wavelengths would scale with the landslide length only, while in the deep-water limit the landslide effectively becomes a point source and the wavelengths would scale with the water depth only. Both are important in the intermediate case, implying that weakly dispersive (or nondispersive) models may not capture all of the physics present in the measured wave fields. 

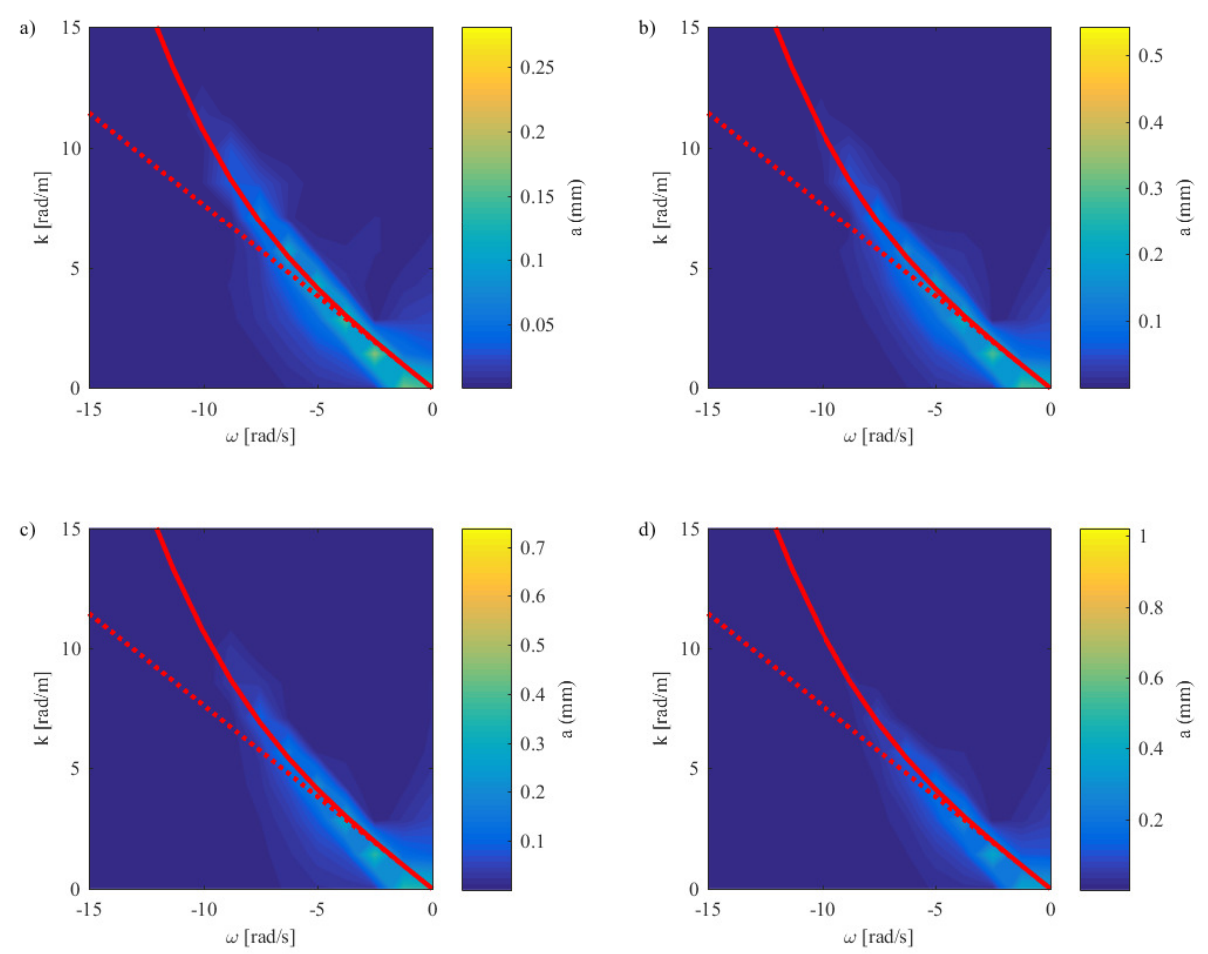

Fig. 9 Two-dimensional wave spectra (from 2D-FFT) for the onshore-propagating waves generated by a landslide with $\lambda=0.153, \tau=0.35$ and a) $F r=0.125$, b) $F r=0.25$, c) $F r=0.375$ and d) $F r=0.5$. The solid line represents the linear dispersion relation and the dotted line represents the shallow-water wave speed.

Increasing the landslide acceleration increased the amplitude of the leading offshore-propagating wave and dispersive wave train (a known result [16]). Although the leading wave approached the shallow-water limit, it still exhibited a lengthening associated with frequency dispersive behaviour (discussed in Section 5). Increasing the leading wave amplitude therefore caused the wave to approach solitary wave-like behaviour, where the dispersive lengthening was (at least partly) balanced by nonlinear steepening of the wave profile. However, even for the highest acceleration value tested during the physical experiments, the leading wave did not have the permanence of form associated with a solitary wave. Although the nonlinearity within the wave field depended primarily on the landslide Froude number, this would also be affected by the landslide acceleration. For shallowwater scenarios with limited constant-velocity periods of motion, the acceleration would govern the nonlinearity of the wave field.

For a given Froude number, a large change in the acceleration could also affect the evolution of the wave field. A reduction in the landslide acceleration would reduce the generated amplitudes and increase the time $\left(t_{1}\right)$ for the landslide to reach its terminal velocity If this time was larger than the characteristic time of wave evolution then (in the extreme case) some of the offshorepropagating waves could pass over the landslide before it reached its terminal velocity, removing the possibility of further energy input from the landslide to those waves. However, the longer time period of energy input from the landslide before its terminal velocity could increase the amplitudes 


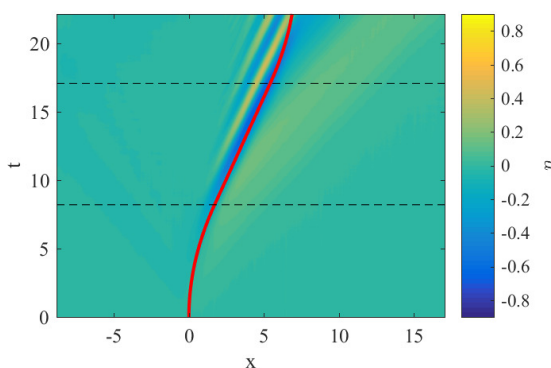

c)
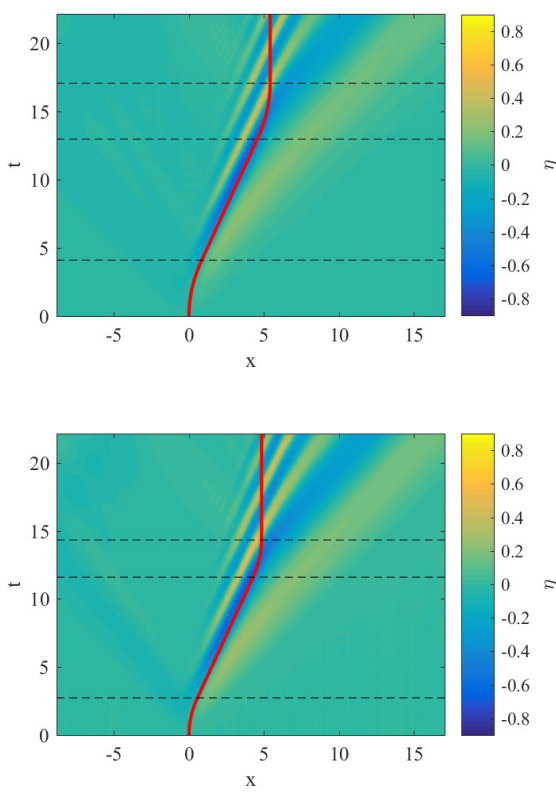

b)

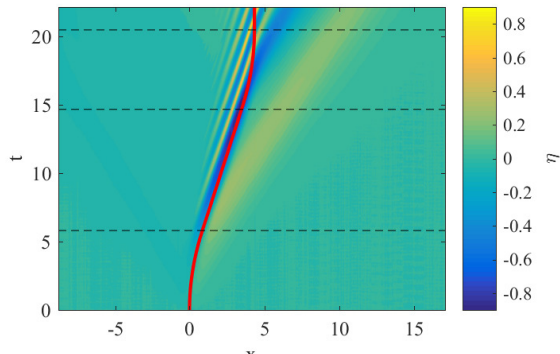

d)
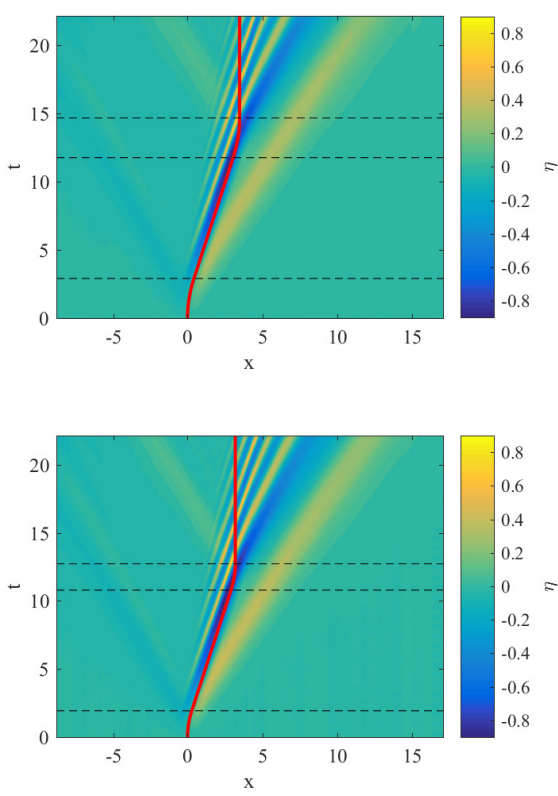

Fig. 10 Comparison between wave fields generated by landslides with a Froude number of 0.5 for different accelerations and submergence depths. Subplots (a) to (f) represent Experiments 1 to 6 in Table $1(\tau=0.70$ and $0.35, \lambda=0.051,0.102$ and 0.153$)$. A solid red line represents the position of the landslide centre of mass, and horizontal dashed lines show the times of motion change.

(hence nonlinearity) of waves propagating near to the landslide. The latter effect is evident in the results presented in Section 6.

These results have implications for our understanding of submerged objects moving at constant velocity. For a given landslide geometry, the water depth controls the dispersive nature of the generated waves. The landslide acceleration controls the initial amplitudes, and hence nonlinearity, of the generated waves. However, lower accelerations increase the time period over which the landslide may input energy into the wave field. It is important to consider this 'pre-conditioning' of the wave field when assessing their subsequent behaviour at constant velocity. Significant variations in 
acceleration may also affect the overall evolution of the wave field. Section 6 discusses the effect of varying landslide accelerations, based on an extended set of numerical simulations.

In the case of a submarine landslide moving along a sloping boundary, the initial acceleration of the landslide would occur in the shallowest water depth. This acceleration would therefore be the most important parameter governing the nonlinearity of the generated waves, as found by Watts [20]. The depth dependence on location would cause the offshore-propagating waves to increase in wavelength and celerity, while the shoaling onshore-propagating waves would increase in amplitude while decreasing in wavelength and celerity. Continuous energy input to the offshore-propagating waves would therefore require the landslide to continue its acceleration indefinitely, rather than approach a terminal velocity, and is therefore less likely to occur in the field.

The results indicate that (for a given landslide terminal velocity) the largest trailing waves may be observed for lower landslide accelerations, and therefore a landslide running out long distances over very shallow slopes is the most likely to generate such waves. Since the trailing waves were relatively short (and hence dispersive), these would be most dangerous in the vicinity of the landslide, in the case where a shoreline was located in the direction of landslide motion. Large bays or fjords may be most at risk from these waves. The leading waves were less dispersive, and so would be dangerous at locations further removed from the source (although these would be unlikely to cross oceans as in the case of earthquake-generated tsunamis). Since their amplitude was governed by the initial acceleration of the landslide, a landslide initiated on a steep slope is likely to be more dangerous for nearby coastal communities.

\subsection{Parametric dependence of potential energy and maximum amplitudes}

This section discusses the parametric dependence of the maximum amplitudes of the generated waves, and the maximum potential energy in the wave field, as a function of the landslide Froude number $F r$, initial landslide acceleration $\lambda$ and submergence depth $\tau$. As in the discussion of the trailing waves, it is useful to extend the parameter space to include lower Froude numbers, drawing on the wave field results from Whittaker et al. [21]. Their experiments identified the landslide Froude number as the dominant parameter affecting the properties of the generated wave field for experiments undertaken between $F r=0.125$ and $F r=0.375$. Shallower submergence depths are known to generate larger wave amplitudes [16], and the effect of landslide acceleration on the wave field properties observed at $F r=0.5$ are discussed in the previous section.

We first consider the maximum amplitudes of the waves generated by the moving landslide. The maximum amplitudes of the generated waves have obvious relevance for communities potentially at risk from landslide-generated tsunamis. Figure 11 shows the dependence of the maximum crest and trough amplitude as a function of the landslide Froude number, combining the $F r=0.5$ experiments listed in Table 1 with the lower- $F r$ results of Whittaker et al. [21]. Note that the results of Experiments 7 and $8(F r=0.625$ and 0.75 , respectively) are not shown, as the largest amplitudes of the generated waves were limited by wave breaking.

The landslide Froude number affected not only the maximum amplitudes of the generated waves, but which wave groups dominated the generated wave field [21]. At lower landslide Froude numbers, the wave field was dominated by the waves generated during the initial acceleration of the landslide. Thus, at low Froude numbers the largest crest was the leading crest propagating in front of the landslide in the offshore direction, while the largest trough was the leading trough propagating in the onshore direction. As the landslide Froude number increases, the free surface depression located above the landslide during its constant-velocity motion became larger than the leading trough propagating in the onshore direction. As the landslide Froude number increased further, the largest crest amplitudes were located in the trailing wave group behind the landslide (also during its constant-velocity motion), rather than in the offshore-propagating group generated by the landslide acceleration. It is expected that the leading waves, the free surface depression and 

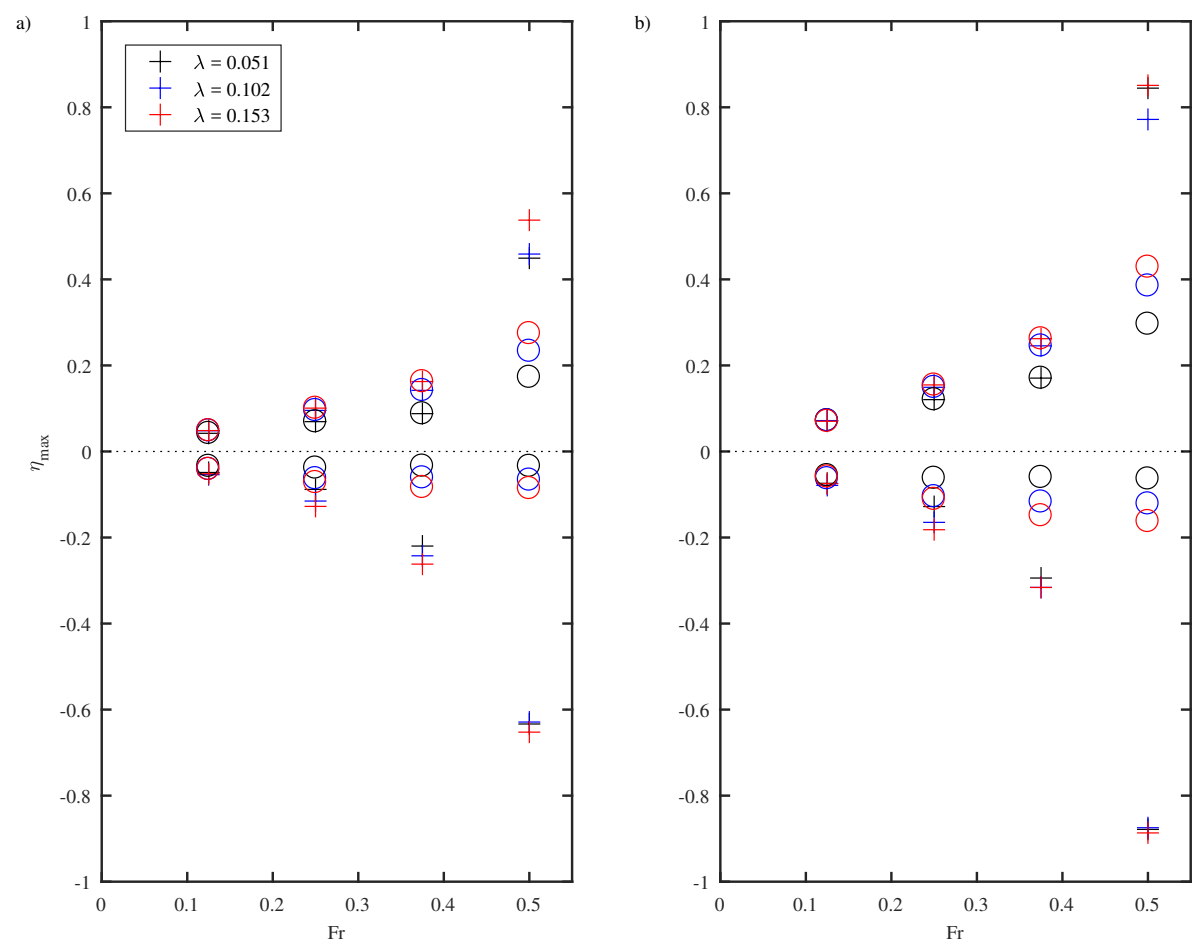

Fig. 11 Dependence of maximum wave amplitude on landslide Froude number and nondimensional landslide acceleration for a) $\tau=0.70$ (deep case), and b) $\tau=0.35$ (shallow case). Note that crosses represent the maximum crest or trough amplitude over the entire wave field, while circles represent the maximum leading crest or trough amplitude. The dashed horizontal line at $\eta=0$ separates the maximum crest and trough amplitudes. $\tau=0.70$ (deep case), and b) $\tau=0.35$ (shallow case).

the trailing waves have different parametric dependences, and so the maximum amplitudes of the leading waves are therefore represented by circles in Figure 11. The maximum amplitudes over the entire generated wave field are represented by crosses.

Figure 11 shows that the maximum crest amplitude was equal to the amplitude of the leading offshore-propagating crest for $F r \leq 0.375$. Only at $F r=0.5$ was the largest crest amplitude located within the trailing wave group propagating behind the landslide during its constant-velocity motion. This behaviour was consistent for all landslide accelerations and submergence depths tested in the physical experiments. The free surface depression above the landslide became larger than the leading onshore-propagating trough at much lower Froude numbers. Although it should be noted that the free surface depression and onshore-propagating trough were difficult to separate at early times within the experiments, the Froude number dependence of the trapped free surface depression and that of the trailing waves clearly differ over the parameter space tested.

The Froude number dependence of the leading crest amplitude was much stronger than that of the leading trough amplitude, since the direction of the landslide motion allowed a longer time period of interaction with the leading crest. However, the amplitude of the leading trough did still exhibit weak dependence on the landslide Froude number, over the range of Froude numbers tested. It is expected that (for a low enough $\lambda$ value) the leading trough amplitude would become effectively 


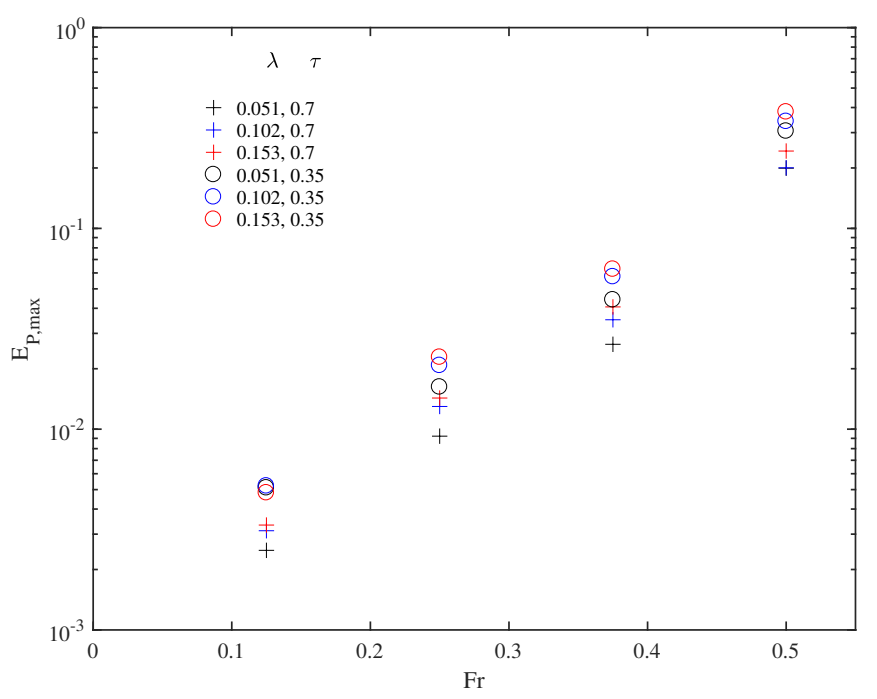

Fig. 12 Maximum potential energy dependence on landslide Froude number, combining the current dataset with results of lower-Froude number experiments conducted in [21].

$F r$ independent at higher $F r$ values. As might be expected, the $\lambda$ dependence of the leading trough was much stronger than that of the trapped free surface depression above the landslide during its constant-velocity motion

The spatial resolution of the predicted and measured free surface elevations allows calculation of the (nondimensional) potential energy within the wave field, according to:

$$
E_{P}(t)=\frac{1}{2} \int_{-\infty}^{\infty} \eta(x, t)^{2} d x
$$

This integrated quantity provides a bulk measure of the energy within the wave field, accounting for the leading waves generated by the initial landslide acceleration, the trapped depression propagating above the landslide during its constant-velocity motion and the trailing waves generated during this constant-velocity motion. Figure 12 shows the variation of the maximum potential energy against the landslide Froude number, considering the experiments shown in Figure 11. The circles represent the shallower submergence depth $\tau=0.35$, while the crosses represent the deeper submergence depth of $\tau=0.7$. At these Froude numbers, it is assumed that the reflection of onshore-propagating waves before the end of the experiment had a negligible effect on the maximum potential energy. The vertical axis of Figure 12 is plotted on a log scale. The maximum potential energy appears to follow a linear trend when plotted in this manner. However, the results presented in Figure 11 show that the wave field moves from a leading-wave dominated regime to a trailing-wave dominated regime at $F r=0.5$.

\section{Model predictions of experimentally generated wave fields}

5.1 Effect of landslide acceleration at $F r=0.5$

We now compare the experimentally-measured wave field to the predictions of the CFD model, using both the laminar and turbulent solvers described in Section 2. The spatial and temporal 

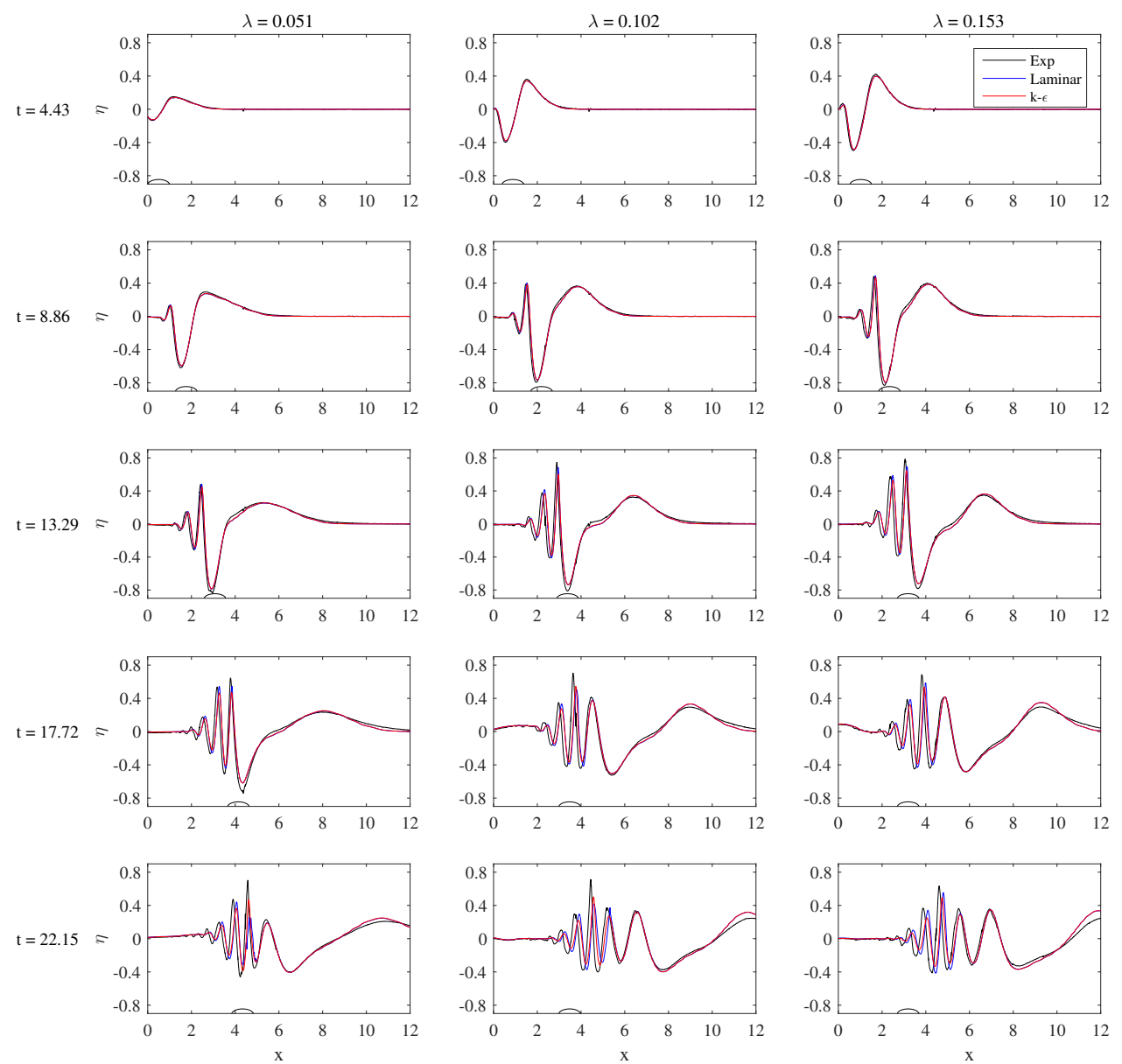

Fig. 13 Comparison of predicted and measured wave propagation in the offshore direction for Experiments 2, 4 and 6 (conducted with $F r=0.5, \tau=0.35$ and $\lambda=0.051,0.102$ and 0.153 ). Variables have been nondimensionalised according to Equation 2. The horizontal landslide location is shown for reference, although its thickness and vertical location are not shown to scale.

resolution of the free surface measurements provides a robust check of the predictive capability of the CFD model. The comparisons are conducted at the shallower submergence depth of $\tau=$ 0.35 , for the three accelerations tested in the physical experiments $(\lambda=0.051,0.102$ and 0.153$)$. Initially the comparisons are conducted at $F r=0.5$. Since the CFD model contains no assumptions regarding the wavelength or amplitude of the generated waves, we expect good agreement between the predicted and measured wave fields.

Figure 13 compares the predicted and measured wave fields in the offshore (positive $x$ ) region at five successive times. Although presented in nondimensional form, these times are equivalent to dimensional times of $1 \mathrm{~s}, 2 \mathrm{~s}, 3 \mathrm{~s}, 4 \mathrm{~s}$ and $5 \mathrm{~s}$ after the initiation of the landslide motion. The horizontal location of the landslide is shown in each image. 
Both laminar and turbulent models accurately captured the waves generated by the initial acceleration of the landslide, with very slight $(\sim 5 \%)$ under-prediction of the maximum amplitude of the generated waves. Although the predicted free surface contained some small-scale sinusoidal perturbations, these were of a similar size to the noise in the experimentally-measured free surface elevations and may be neglected. The acceleration phase of motion ceased at $t=5.80,2.90$ and 1.94 for the three accelerations $\lambda=0.051,0.102$ and 0.153 respectively. Thus, the landslide had already begun its constant-velocity motion in two of the three wave field plots shown at $t=$ 4.43. The agreement between model and experiment appears excellent at this time for all of the landslide accelerations tested. The laminar and turbulent solutions were indistinguishable at this time, demonstrating that both grid resolution (see Section 2) and the inclusion of a turbulence model have a negligible effect on the CFD model's ability to simulate the relatively long leading waves.

The agreement between the predicted and measured waves reduced slightly during the constantvelocity phase of landslide motion. The leading wave measured in the experiments, which exhibited minimal interaction with the landslide after its initial generation, reduced in amplitude more rapidly than the predicted leading wave (again, the laminar and turbulent leading waves were identical). Indeed, at $t=17.72$ the amplitude of the predicted leading wave was larger than the measured leading wave, despite the larger initial amplitude of the measured wave. Based on the convergence results of Section 2 and the excellent agreement between the laminar and turbulent models for the leading wave, the experimental/numerical discrepancy may be caused by departures of the experimental setup from the idealised model geometry. These departures are discussed later in this section.

The discrepancies between both models and the experiment results were most pronounced within the dispersive wave train propagating behind the landslide during its constant-velocity motion. The differences between the laminar and turbulent model results were also most evident in these trailing waves. Although the phase agreement between the simulated and measured free surface depression (which propagated above the landslide) was excellent, the measured trailing waves appeared to lag slightly behind the predicted waves, with the phase discrepancy growing over the remainder of the experiment. The measured waves also had larger amplitudes and slightly longer wavelengths than the predicted waves, although it should be noted that the amplitude agreement is far better than would be obtained using a linear wave model (see Whittaker et al. [21]). The longer wavelengths may be at least partially explained by the slotted false floor used in the experiments. An increase in effective depth due to this slot may have increased the wavelength of those experimentally-measured waves satisfying the $c_{p}=u_{t}$ condition for ongoing energy input from the landslide (discussed in relation to Figures 6 and 7). Other possible effects of the slotted false floor are discussed later in this section.

Despite their lower amplitudes, the waves predicted by the laminar solver exhibited greater nonlinear steepening than both the measured waves and the waves predicted by the turbulent solver. This steepening led to breaking of the first trailing wave in the low-acceleration $(\lambda=0.051)$ simulation by the laminar solver, which was not reproduced in the turbulent model predictions or the experimental measurements. It should be noted that this breaking was observed at several grid resolutions within the laminar model. Although the waves predicted by the laminar solver became very steep for the higher $\lambda$ values, the predicted waves did not break at the higher accelerations. The additional dissipation of the turbulent model was responsible for generally lower amplitudes (of both crests and troughs) predicted by the turbulent model, compared to the laminar model predictions. Although this additional dissipation meant that the under-prediction of the experimentally-measured wave amplitudes by the laminar model was even more pronounced for the turbulent model, the turbulent model more accurately reproduced the shapes of the trailing waves. The turbulent model also correctly predicted the relative amplitudes of the different waves within the trailing wave group, which the laminar model failed to achieve. This indicates that a 
$k-\epsilon$ model may provide non-conservative estimates of landslide-generated waves, but that it will predict the shapes and phasing of these waves more effectively than a laminar CFD solver.

The potential energy in the wave field provides an effective check on the agreement between the predicted and measured wave fields. Figure 14 compares the time-dependent potential energy for the three nondimensional accelerations shown in Figure 13. In terms of parametric dependence, the potential energy was more affected by the larger-amplitude leading waves than by the steeper trailing waves. Thus, the larger amplitudes of the leading waves generated by the higher landslide accelerations had the greatest impact on the maximum potential energy within the wave field, even if these high accelerations did not lead to the steepest trailing waves.

Both models clearly under-predicted the amount of energy within the wave field, including during the initial acceleration of the landslide. Although the phase agreement between the predicted and measured leading waves was excellent, both models under-predicted the amplitudes of the leading crest and trough generated by the initial acceleration of the landslide. Two possible reasons for this consistent amplitude under-prediction are the adoption of $\alpha=0.5$ as the free surface location within the VOF solver or the deformed grid used to simulate the semi-elliptical landslide in the CFD solver. A lower value of $\alpha$ would locate the free surface closer to the 'completely dry' cell, and could contribute to better agreement with the experimental data. Alternatively, vertically integrating the $\alpha$ parameter at each horizontal location may yield better results (this may also improve the model convergence). In terms of the deformed grid, Figure 2 shows that the vertical front and rear faces of the landslide block could not be fully resolved. This smoothing of the landslide shape may have decreased the amplitudes of the waves predicted by both models.

The wave breaking observed in the laminar results for $\lambda=0.051$, shown in Figure 13, caused a decrease in the potential energy within the wave field predicted by the laminar model. This decrease is clearly visible in the top panel of Figure 14, where the blue line (laminar model) crosses the red line (turbulent model) at approximately $t=21$. Some spilling may have occurred in the the trailing waves within the laminar prediction of the $\lambda=0.102$ experiment, based on a slight decrease in the potential energy within the middle panel of Figure 14. The $\lambda=0.153$ results show no such decreases in the potential energy within the wave field predicted by the laminar model. Indeed, the turbulent model potential energy exhibited more of a decrease than the laminar model potential energy in the bottom panel of Figure 14, which may be attributed to the greater dissipation in the $k-\epsilon$ model. The attenuation of energy within the physical experiments may be at least partially due to sidewall friction (discussed below). Friction at the end boundaries of the laboratory flume may have also dissipated some energy in the experimentally-measured wave field. Since the measurement gantry could not reach the extreme ends of the experimental wave flume, slight phase discrepancies between incident and reflected waves at the ends of the flume may have contributed to some of the fluctuations in the energy within the experimentally-measured wave field; these fluctuations were entirely absent from the energy in the predicted wave fields.

Although the laminar and turbulent CFD models presented in this paper do not have the applicability limits of (say) a shallow-water or linear wave theory model, there are several differences between the idealised models and the experiments that may help to explain some of the discrepancies shown in Figures 13 and 14. The experimental wave flume was $250 \mathrm{~m}$ in width, and the effects of sidewall boundary layers on the generated waves would not have been replicated within the twodimensional CFD solver. As discussed in Section 3, the laminar CFD model essentially functioned as a laminar Navier-Stokes solver in the absence of a turbulence model (or resolution of fine eddy scales). However, the turbulent model still contained amplitude and phase discrepancies with the experimentally-measured trailing wave group. A more serious issue with the numerical model is that the VOF (volume of fluid) scheme is slightly diffusive. These effects are accumulative and spatially varying, making them difficult to quantify. Numerical diffusion could account for some of the discrepancies between the predicted and measured wave fields.

In addition to these considerations, the experimental setup contained some departures from the idealised geometry used by the numerical model. The mechanical system providing the landslide 

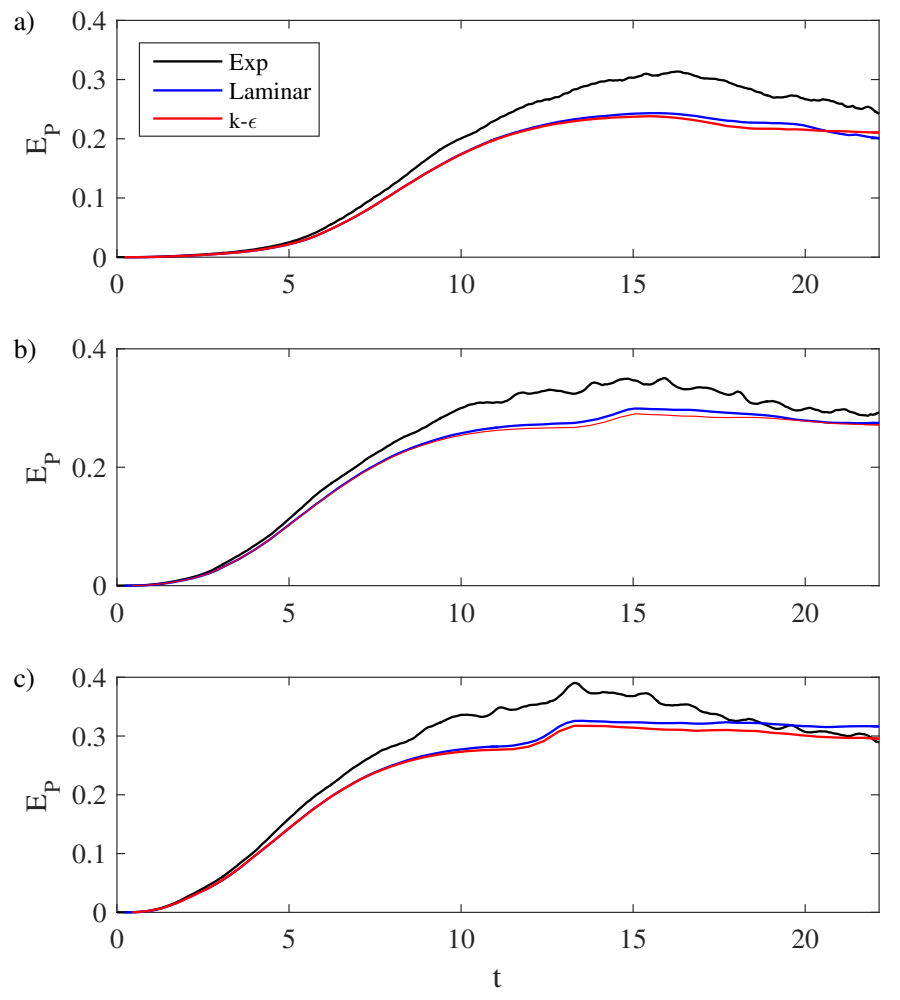

Fig. 14 Time dependence of (nondimensional) potential energy within the experimentally-measured and numerically-predicted wave fields for a landslide Froude number of 0.5 , nondimensional water depth of $\tau=0.35$ and nondimensional accelerations of a) $\lambda=0.051$, b) $\lambda=0.102$ and c) $\lambda=0.153$.

motion was connected to the landslide through a slot of $8 \mathrm{~mm}$ width in the false floor (see Figure 1). This creates the possibility of fluid exchange through the slot, particularly during the constantvelocity phase of motion. This exchange flow would be three-dimensional in nature, and may have slightly altered the amplitudes of the generated waves (particularly in the vicinity of the flume centreline). A thin layer of fluid could also flow between the landslide and the false floor itself, although guides were installed at the front and rear of the landslide block to prevent lifting at high velocities. Another possible consequence of the slot is the leakage of energy to the fluid beneath the false floor, due to the pressure differential over the false floor. The slot was $5 \mathrm{~m}$ in length, such that the experimental waves might be slightly deformed during their propagation between $x=0$ and $x=10$ (in nondimensional form). This may also account for the slight 'shift' in the $k-\omega$ spectra away from the dispersion relation for the offshore-propagating wave results presented in Figure 8.

The laminar and turbulent models captured the phasing of the leading waves very well, although they both under-predicted the amplitudes of these leading waves (and hence the energy within the wave field). Although the experimental sources of dissipation should be greater than the numerical sources (particularly in the case of the laminar model), these amplitude under-predictions were consistently observed over the parameter space. The agreement between the predicted and measured wave fields worsened during the constant-velocity phase of motion. Here the differences between the laminar and turbulent solvers became more pronounced, and the turbulent model per- 
formed better in predicting the shapes and relative amplitudes of the measured waves (although its absolute amplitude under-predictions were slightly worse than those of the laminar model). The premature breaking of excessively steep waves was only observed in the laminar model. However, the 'smoothing out' of this breaking in the turbulence model led to phase shifts in the steepest of the trailing waves.

\subsection{Higher Froude number behaviour}

Figure 15 shows the offshore wave fields for the experiments conducted at Froude numbers of 0.625 (Experiment 7) and 0.75 (Experiment 8). At these Froude numbers, the first of the trailing waves (propagating behind the landslide in the offshore direction) broke during the physical experiments (consistent with the observations of Lee et al. [9] and the model results in the previous section). Any air entrained within the broken wave would not be detected within the LIF measurements, which identify the maximum vertical pixel containing water within the image irrespective of whether lower pixels contain only water or a water-air mixture (similar to the VOF approach of the numerical model, see Section 3). The localised effects of the breaking wave did not greatly affect the overall group structure, apart from those waves in the immediate vicinity of the breaking wave.

The wave breaking appeared to occur earlier within the laminar model than in the physical experiments. This is consistent with the comparisons at $\lambda=0.051$ shown in Figure 13, where the predicted breaking of the first of the trailing waves in the laminar model was not observed within the physical experiments. The possible reasons for this early onset of breaking, and other discrepancies between the predicted and measured wave fields, are discussed in the previous section. Although the turbulent model appeared to provide an accurate representation of the unbroken wave shapes within the experimentally-measured wave field, its prediction of the broken wave shapes appeared to be quite poor (even making allowances for the issues with the LIF method for broken waves). This is clearly visible at $t=13.29$. However, unlike the laminar model, the turbulent model did not predict the onshore propagation of a small-amplitude group of short waves released by the breaking wave. Thus, the dissipation of the $k-\epsilon$ model may have advantages and disadvantages when predicting the properties of broken waves. The assumed incompressibility of air in the CFD models is likely to have negatively affected the predictions of both laminar and turbulent solvers.

\section{Effect of landslide acceleration - extended numerical simulations}

A major advantage of numerical models is their ability to test a broader parameter space than can be replicated within physical experiments. Considering the effect of the landslide acceleration on the properties of the generated waves, it is useful to investigate the low- and high-acceleration limits of the parameter space. Three additional numerical simulations were performed with the laminar and turbulent models at $\lambda$ values of $0.045,0.5$ and 1.0 respectively, to assess the effect of larger variations in landslide acceleration (in particular) on the wave field evolution. The landslide Froude number was fixed at 0.5 in all of these simulations. The low-acceleration limit was governed by the maximum test duration, as a landslide with an extremely low acceleration would not complete its acceleration within the experimental time limit of $t=22.15$, or $5 \mathrm{~s}$ in dimensional terms.

Figure 16 illustrates the overall evolution of the wave field for the three numerical simulations. The parametric dependence is consistent with Figure 6, where the offshore- and onshorepropagating waves generated by the initial landslide motion were relatively larger for higher $\lambda$ values. However, at this Froude number the waves generated by the constant-velocity phase of motion dominated the wave field at later times (see Whittaker et al. [21] for examples of accelerationdominated wave fields at lower Froude numbers). Indeed, the onshore-propagating waves are barely visible for the lowest- $\lambda$ simulation. The almost identical wave fields generated by the two larger 

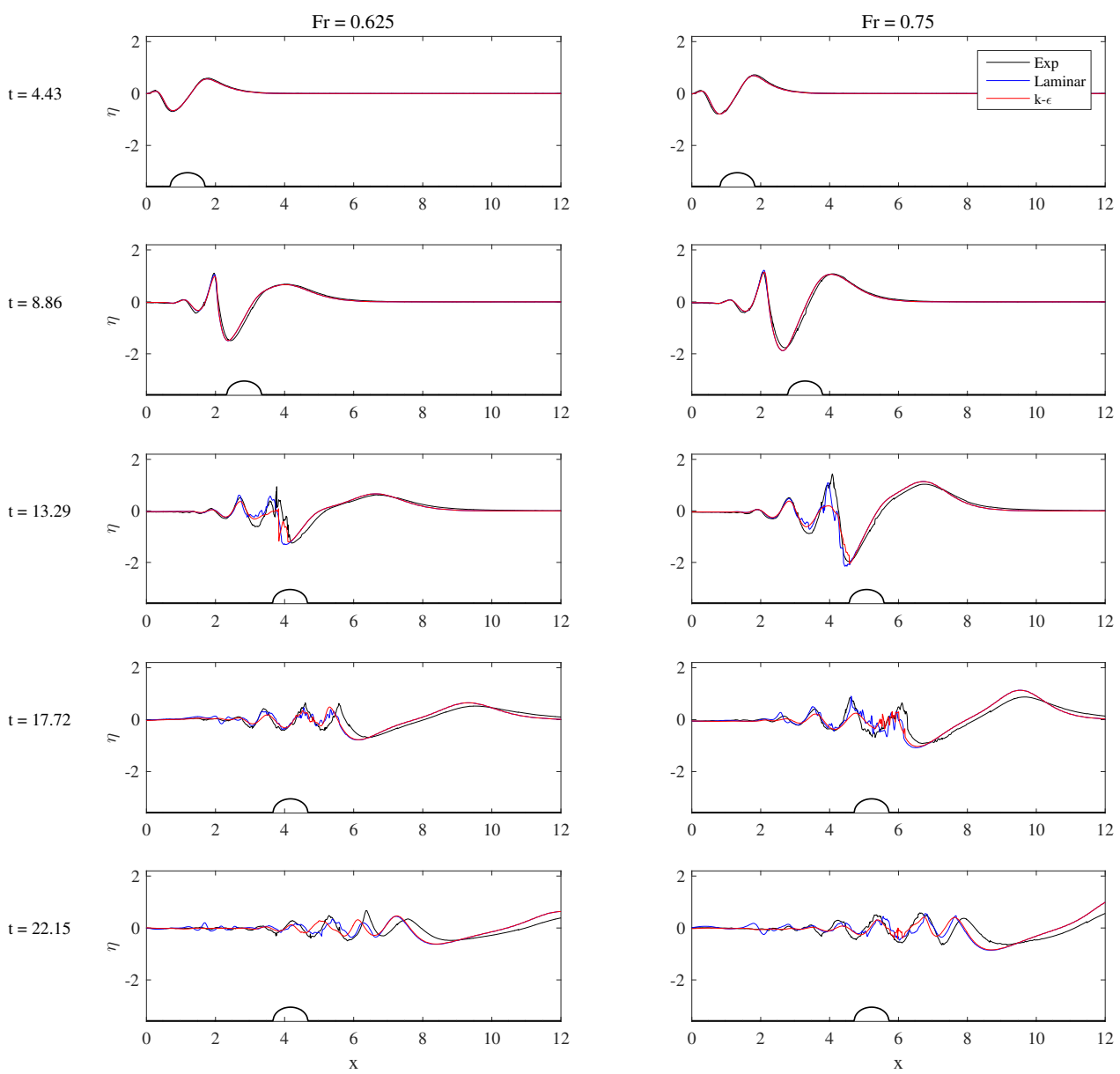

Fig. 15 Measured and predicted free surface elevations from Experiments 7 and 8, with Froude numbers of 0.625 and 0.75 respectively $(\tau=0.35, \lambda=0.153)$.

accelerations suggest that a landslide acceleration of approximately $\lambda=1$ may be considered to be the 'instantaneous acceleration' limit for this landslide Froude number. This limit is likely to be lower for smaller landslide Froude numbers.

Figure 17 compares the offshore-propagating waves generated by the three landslide accelerations, as predicted by the laminar and turbulent models. In all cases, the amplitudes of the short crests and troughs were larger in the predictions of the laminar model than the turbulent model. However, the phasing of the waves predicted by the laminar and turbulent models were essentially identical. For the larger two landslide accelerations, increasing $\lambda$ by a factor of 2 only resulted in a small phase shift of the generated waves. Increasing the landslide acceleration led to larger leading waves, as would be expected. However, an interesting feature of this figure is that the nonlinearity of the trailing waves appeared to decrease with increasing landslide acceleration. Wave breaking occurred in the laminar $\lambda=0.045$ simulation at $t=21.70$ (just prior to the end of the simulation), 

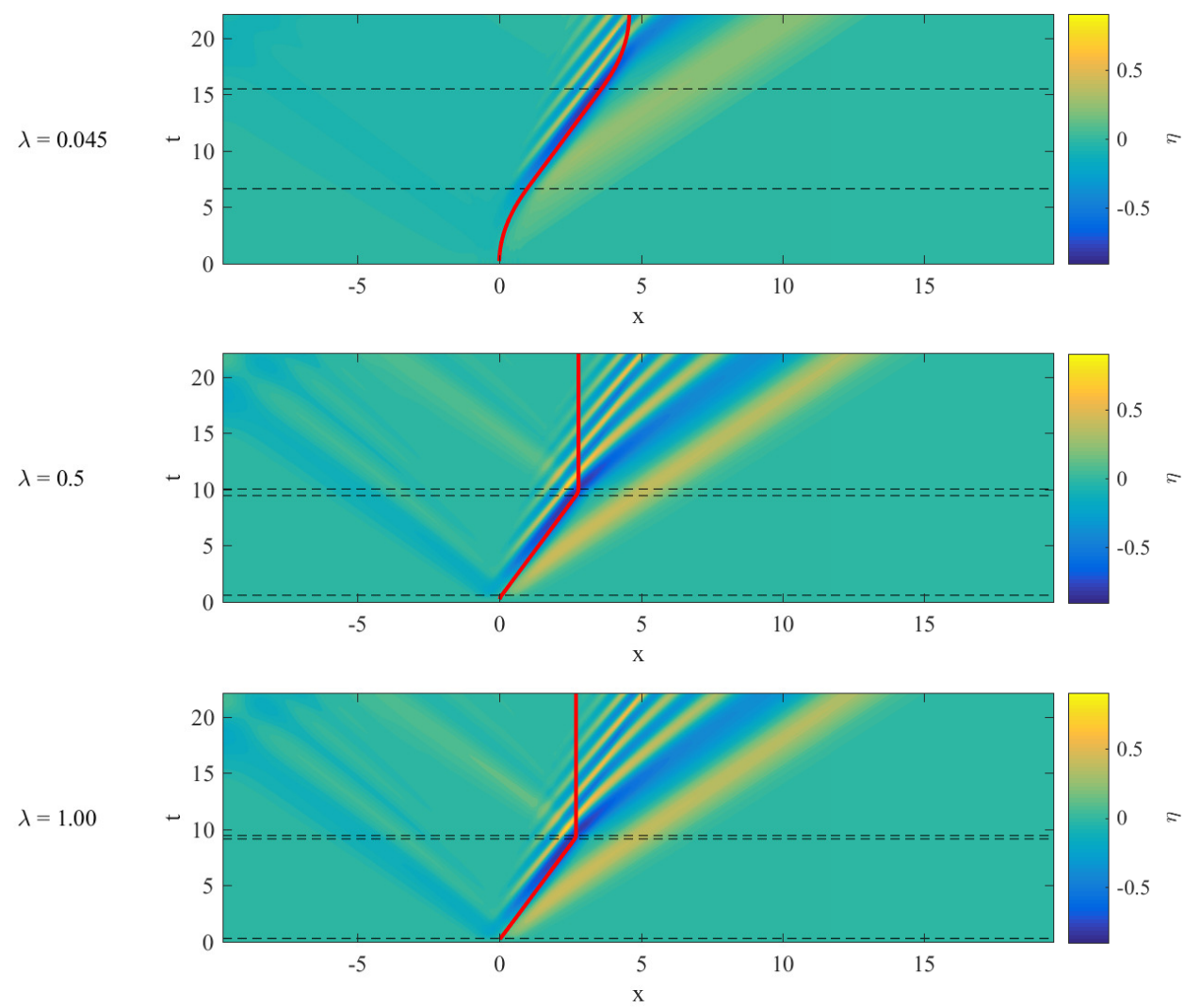

Fig. 16 Effect of landslide acceleration on the wave field evolution for numerical simulations conducted with $\tau=0.35$ and $F r=0.5$. The solid red line shows the location of the landslide centre of mass, while the horizontal dashed black lines show the times of motion change.

and a reduction in nonlinearity of the trailing waves is apparent between the $\lambda=0.5$ and $\lambda=1.0$ simulations (which did not appear to contain any breaking). This wave breaking was not observed in the wave field predicted by the turbulent model.

These results have implications for previous experimental or numerical studies that have either considered the waves generated by the acceleration or terminal velocity of a submerged landslide (or generic submerged object) in isolation. As shown by Whittaker et al. [21], the landslide Froude number is a good indicator of whether the initial acceleration or terminal velocity will govern the evolution of the wave field. However, the trailing wave results demonstrate that even a localised group of waves dominated by the landslide terminal velocity can be significantly modified by the landslide's acceleration to that terminal velocity. The assumption of an instantaneous acceleration to terminal velocity may be inappropriate for some cases, and indeed appears to underestimate the nonlinearity of the trailing waves in the vicinity of the landslide (despite maximising the leading waves). Both acceleration and terminal velocity should be included as key parameters when considering the waves generated by the motion of a submerged body. 

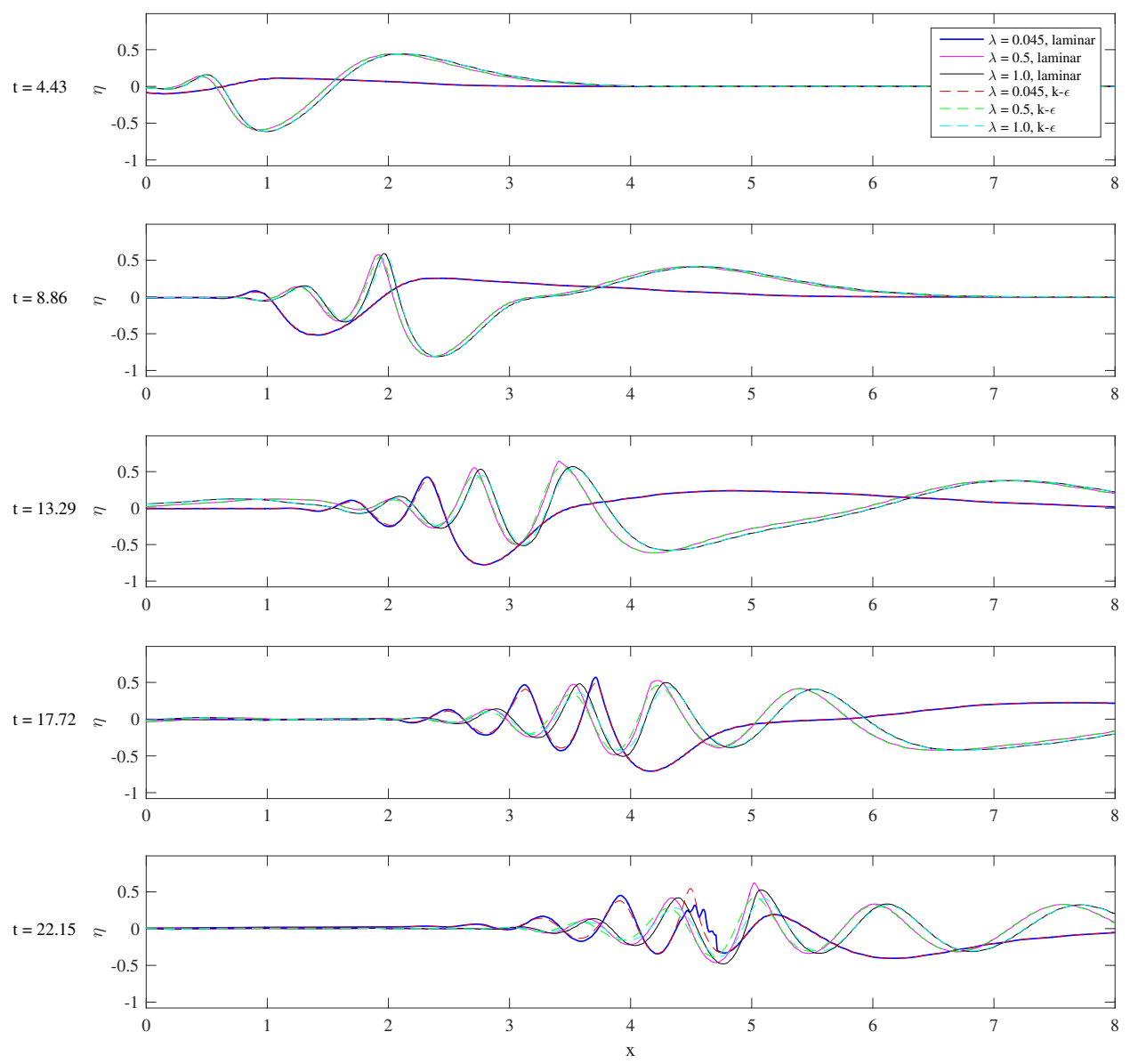

Fig. 17 Comparison between the offshore-propagating waves generated by a landslide with $\tau=0.35$ and $F r=0.5$, from the extended numerical simulations.

\section{Conclusions}

The experimental system of Whittaker et al. [21] allowed accurate control of landslide motion for Froude numbers of up to 0.75 over a range of landslide accelerations, in addition to accurately resolving spatial and temporal variations in free surface elevation. These experiments enabled an investigation into the energy transferred from the landslide to the offshore-propagating wave packet during its constant-velocity motion, and provide a robust dataset for numerical model validation.

A 2D-FFT of the experimental wave field demonstrated the trapping of wave energy at the landslide velocity, modifying the dispersion relation of those waves. This effect was only observed in the offshore region of the wave field, and increased in significance with increasing landslide Froude number. The crest amplitudes within the trailing wave group became larger than the leading crest 
amplitude for $F r=0.5$, while the trapped free surface depression became larger than the leading onshore-propagating trough at much lower Froude numbers.

The IHFOAM CFD package [8] accurately captured the general behaviour of the waves generated by the initial acceleration of the landslide. Both laminar and turbulent models under-predicted the amplitudes of the experimentally-measured waves. The most pronounced phase and amplitude discrepancies were observed for the group of short trailing waves, where the waves predicted by the models were also shorter than the measured waves. The waves predicted by the laminar model steepened and broke before the waves predicted by the turbulent model or measured during the experiments. In some cases, only the waves predicted by the laminar model broke.

Both models consistently under-predicted the potential energy within the experimentally-generated wave field over the parameter space investigated. Possible reasons for the discrepancies include numerical diffusion by the VOF solver, the landslide approximation in the numerical model, the definition of the free surface within the VOF solver, the effects of the slotted false floor and fluid flow beneath the experimental landslide block. The turbulence model was more effective at capturing the shapes of the experimentally-generated waves, and thus the breaking (or lack thereof) more closely coincided with the experiments.

The model predictions over an extended parameter space show the importance of considering the initial landslide acceleration, even at Froude numbers where the wave field is dominated by interactions with the landslide during its constant-velocity motion. For otherwise identical experiments, the leading waves had larger amplitudes (and hence greater nonlinearity) for larger magnitudes of the landslide acceleration. However, the trailing waves generated by lower landslide accelerations were more nonlinear than those generated by higher landslide accelerations. This is important to consider, and experiments concerning submerged objects moving at constant velocities should not necessarily assume an instantaneous initial acceleration to the constant velocity.

Acknowledgements The laboratory work in this project was conducted with the assistance of Ian Sheppard, Kevin Wines, Alan Stokes and Mike Weavers. Prof. Liu would like to acknowledge the support received from Cornell University and National University of Singapore. The provision of IHFOAM by Dr. P. Higuera is also greatly appreciated, as are contributions by Pedro Lee and Sarah Delavan.

\section{References}

1. Dean, R.G., Dalrymple, R.A.: Water wave mechanics for engineers and scientists. (1991)

2. Di Risio, M., De Girolamo, P., Bellotti, G., Panizzo, A., Aristodemo, F., Molfetta, M.G., Petrillo, A.F.: Landslide-generated tsunamis runup at the coast of a conical island: New physical model experiments. J. Geophys. Res. 114(C1), C01,009 (2009)

3. Enet, F., Grilli, S.T.: Experimental study of tsunami generation by three-dimensional rigid underwater landslides. Journal of Waterway, Port, Coastal, and Ocean Engineering 133(6), 442-454 (2007)

4. Fritz, H.M., Hager, W.H., Minor, H.E.: Landslide generated impulse waves. Experiments in Fluids 35(6), 505-519 (2003)

5. Fritz, H.M., Hager, W.H., Minor, H.E.: Landslide generated impulse waves. 2. hydrodynamic impact craters. Experiments in Fluids 35(6), 520-532 (2003)

6. Fritz, H.M., Hager, W.H., Minor, H.E.: Near field characteristics of landslide generated impulse waves. Journal of Waterway, Port, Coastal, and Ocean Engineering 130(6), 287-302 (2004)

7. Hampton, M.A., Lee, H.J., Locat, J.: Submarine landslides. Reviews of Geophysics 34(1), 33-59 (1996). DOI 10.1029/95RG03287

8. Higuera, P., Losada, I.J., Lara, J.L.: Three-dimensional numerical wave generation with moving boundaries. Coastal Engineering 101, 3547 (2015). DOI 10.1016/j.coastaleng.2015.04.003

9. Lee, S.J., Yates, G.T., Wu, T.Y.: Experiments and analyses of upstream-advancing solitary waves generated by moving disturbances. Journal of Fluid Mechanics 199, 569-593 (1989)

10. Lighthill, J.: Waves in Fluids. Cambridge University Press (1978)

11. Liu, P.L.F., Wu, T.R., Raichlen, F., Synolakis, C.E., Borrero, J.C.: Runup and rundown generated by threedimensional sliding masses. Journal of Fluid Mechanics 536(-1), 107-144 (2005)

12. Locat, J., Lee, H.J.: Submarine landslides: Advances and challenges. Canadian Geotechnical Journal 39(1), 193-212 (2002) 
13. Lynett, P., Liu, P.L.F.: A numerical study of the run-up generated by three-dimensional landslides. J. Geophys. Res. 110(C3), C03,006 (2005)

14. Renzi, E., Sammarco, P.: Landslide tsunamis propagating around a conical island. Journal of Fluid Mechanics 650(-1), 251-285 (2010)

15. Sammarco, P., Renzi, E.: Landslide tsunamis propagating along a plane beach. Journal of Fluid Mechanics 598(-1), 107-119 (2008)

16. Sue, L., Nokes, R., Davidson, M.: Tsunami generation by submarine landslides: comparison of physical and numerical models. Environmental Fluid Mechanics 11(2), 133-165 (2011)

17. Sue, L.P.: Modelling of tsunami generated by submarine landslides. Ph.D. thesis, University of Canterbury (2007)

18. Tinti, S., Bortolucci, E.: Energy of water waves induced by submarine landslides. Pure and Applied Geophysics 157(3), 281-318 (2000). URL http://dx.doi.org/10.1007/s000240050001

19. Vennell, R.: Resonance and trapping of topographic transient ocean waves generated by a moving atmospheric disturbance. Journal of Fluid Mechanics 650, 427-442 (2009)

20. Watts, P.: Tsunami features of solid block underwater landslides. Journal of Waterway, Port, Coastal, and Ocean Engineering 126(3), 144-152 (2000)

21. Whittaker, C., Nokes, R., Davidson, M.: Tsunami forcing by a low froude number landslide. Environmental Fluid Mechanics 15(6), 1215-1239 (2015) 